\title{
Determinacy and Subsumption for Single-valued Bottom-up Tree Transducers
}

\author{
Kenji Hashimoto ${ }^{1}$, Ryuta Sawada ${ }^{2}$, Yasunori Ishihara ${ }^{2}$, \\ Hiroyuki Seki ${ }^{1}$, and Toru Fujiwara ${ }^{2}$ \\ 1 Nara Institute of Science and Technology, Japan \\ $\{k$-hasimt, seki\}@is.naist.jp \\ ${ }^{2}$ Osaka University, Japan \\ \{ishihara, fujiwara\}@ist.osaka-u.ac.jp
}

\begin{abstract}
This paper discusses the decidability of determinacy and subsumption for tree transducers. For two tree transducers $T_{1}$ and $T_{2}, T_{1}$ determines $T_{2}$ if the output of $T_{2}$ is identified by the output of $T_{1}$, that is, there is a partial function $f$ such that $\llbracket T_{2} \rrbracket=f \circ \llbracket T_{1} \rrbracket$ where $\llbracket T_{1} \rrbracket$ and $\llbracket T_{2} \rrbracket$ are tree transformation relations induced by $T_{1}$ and $T_{2}$, respectively. Also, $T_{1}$ subsumes $T_{2}$ if $T_{1}$ determines $T_{2}$ and the partial function $f$ such that $\llbracket T_{2} \rrbracket=f \circ \llbracket T_{1} \rrbracket$ can be defined by a transducer in a designated class that $T_{2}$ belongs to. In this paper, we show that determinacy is decidable for single-valued linear extended bottom-up tree transducers as the determiner class and single-valued bottom-up tree transducers as the determinee class. We also show that subsumption is decidable for these classes.
\end{abstract}

\section{Introduction}

In data transformation, it is desirable that certain information in source data be preserved through transformation. As a formalization for information preservation in data transformation, the notions of deteminacy and subsumption (or query rewriting) are known [1-3]. Let $Q$ be a query to a database and $V$ be a data transformation (or a view definition) of the database. Determinacy of $Q$ by $V$ means that the answer to $Q$ is identified by the answer to $V$. When information to be preserved is specified by a query $Q$, determinacy guarantees that for any database instance $D, V(D)$ gives enough information to uniquely determine the specified information $Q(D)$ for $D$. Subsumption means that the answer to $Q$ can always be computed from the answer to $V$ by some query in a designated class that $Q$ belongs to. Compared with determinacy, subsumption guarantees that the necessary information $Q(D)$ can be extracted from the transformed data $V(D)$ by the same query language expressing $Q$.

We study the decidability of determinacy and subsumption when both a query and a data transformation are given by tree transducers. Tree transducers are machines that model relations between labeled ordered trees. A tree transducer is said to be singlevalued if the tree transformation induced by the transducer is a partial function. Since an XML document has a tree structure, tree transducers are often used as a model of XML document transformations. Formally, for two single-valued tree transducers $T_{1}$ and $T_{2}$ in classes $\Pi_{1}$ and $\Pi_{2}$ of transducers, respectively, we say $T_{1}$ determines $T_{2}$ if there is a partial function $f$ such that $\llbracket T_{2} \rrbracket=f \circ \llbracket T_{1} \rrbracket$ (see Fig. 1(a)), where $\llbracket T_{1} \rrbracket$ and 


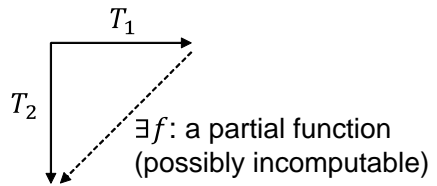

(a) $T_{1}$ determines $T_{2}$

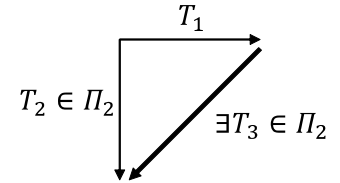

(b) $T_{1}$ subsumes $T_{2}$ w.r.t. $\Pi_{2}$

Fig. 1. Determinacy and subsumption

$\llbracket T_{2} \rrbracket$ are the tree transformation relations induced by $T_{1}$ and $T_{2}$, respectively. $\Pi_{1}$ and $\Pi_{2}$ are called the determiner class and the determinee class, respectively. We also say $T_{1}$ subsumes $T_{2}$ with respect to $\Pi_{2}$, if $T_{1}$ determines $T_{2}$ and the partial function $f$ such that $\llbracket T_{2} \rrbracket=f \circ \llbracket T_{1} \rrbracket$ can be defined by a transducer in the class $\Pi_{2}$ (see Fig. 1(b)). Our goal is to find practical subclasses of tree transducers for which determinacy and subsumption are decidable, and to consider the problem of constructing a tree transducer $T_{3}$ in the determinee class such that $\llbracket T_{2} \rrbracket=\llbracket T_{3} \rrbracket \circ \llbracket T_{1} \rrbracket$ if $T_{1}$ subsumes $T_{2}$.

In this paper, we first show that determinacy is decidable for single-valued linear extended bottom-up tree transducers ( $s l$-xbots) as the determiner class and single-valued bottom-up tree transducers ( $s$-bots) as the determinee class running over a ranked-tree encoding of the given XML document. Transformations induced by transducers in the classes include simple filterings, relabelings, insertions, and deletions of elements. Especially, $s l$-xbots do not allow duplications of elements. Given an $s l$-xbot $T_{1}$ and an $s$-bot $T_{2}$, the decision procedure works as follows: (1) construct a transducer $T_{1}^{i n v}$ that induces the inverse of $T_{1}$, (2) construct a transducer $T_{3}$ that induces the composition of $T_{1}^{i n v}$ followed by $T_{2}$, then (3) decide whether $T_{3}$ is single-valued. We introduce a class of transducers with grafting, which allows to insert any tree in a specified tree language, in order to capture inverses of transformations induced by $s l$-xbots and the composition of the inverses and $s$-bots. Next, we prove that single-valuedness for the class is decidable. For some other classes, we show that determinacy is undecidable even for homomorphism tree transducers as the determiner class, which is a proper subclass of $s$-bots and single-valued top-down tree transducers ( $s$-tops). Moreover, determinacy is undecidable for deterministic monadic second-order logic defined tree transducers (dmsott) $[4,5]$ as the determiner class, which form a class incompatible with $s$-bots and $s$-tops but is a proper superclass of $s l$-xbots. Lastly, we show that subsumption is decidable for $s l$-xbots as the determiner class and $s$-bots as the determinee class. The proof gives a construction method of an $s$-bot $T_{3}$ satisfying $\llbracket T_{2} \rrbracket=\llbracket T_{3} \rrbracket \circ \llbracket T_{1} \rrbracket$ if $T_{1}$ subsumes $T_{2}$.

Related Work. Determinacy and subsumption (or query rewriting) have been well studied mainly for relational queries such as first order logic and conjunctive queries [13]. In XML context, query preservation [6] has been studied as a notion of information preservation of XML mappings. Let $\mathcal{L}$ be an XML query language. For queries $Q, Q^{\prime} \in \mathcal{L}$ and a view $V, V$ preserves $Q$ with $Q^{\prime}$ if $Q=Q^{\prime} \circ V$. This definition is essentially the same as the definition of subsumption. A view $V$ is query preserving with 
respect to $\mathcal{L}$ if there exists a computable function $R_{w}$ such that for any query $Q \in \mathcal{L}$, $V$ preserves $Q$ with $R_{w}(Q)$. Unfortunately, it is known to be undecidable to decide whether $V$ is query preserving with respect to projection queries, given a view $V$ in any query class $\mathcal{L}_{f}$ which can simulate first order logic queries, such as XQuery and XSLT. It is also undecidable whether $V$ preserves $Q$ with some projection query, given $V$ in the class $\mathcal{L}_{f}$ and a projection query $Q$. As far as we know, the decidability of query preservation for other subclasses of XQuery and XSLT has been little investigated.

\section{Preliminaries}

\subsection{Trees and Tree Automata}

We treat only ranked labeled ordered trees and tree transducers which work on such trees. Though an XML document is often modeled by an unranked labeled ordered tree, we assume that an unranked tree is encoded to a ranked tree by some encoding such as First-Child-Next-Sibling encoding [7] and DTD-based encoding [8].

We denote the set of nonnegative integers by $\mathbb{N}$. Let $[i, j]=\{d \in \mathbb{N} \mid i \leq d \leq j\}$. In particular, we denote $[1, k]$ by $[k]$. A (ranked) alphabet is a finite set $\Sigma$ of symbols with a mapping rk from $\Sigma$ to $\mathbb{N}$. We denote the set of $k$-ary symbols of $\Sigma$ by $\Sigma^{(k)}=$ $\{\sigma \in \Sigma \mid \operatorname{rk}(\sigma)=k\}$. The set $\mathcal{T}_{\Sigma}$ of ranked trees over an alphabet $\Sigma$ is the smallest set $T$ such that $\sigma\left(t_{1}, \ldots, t_{k}\right) \in T$ for every $k \in \mathbb{N}, \sigma \in \Sigma^{(k)}$, and $t_{1}, \ldots, t_{k} \in T$. If $\sigma \in \Sigma^{(0)}$, we write $\sigma$ instead of $\sigma()$. The set of positions of $t=\sigma\left(t_{1}, \ldots, t_{k}\right) \in \mathcal{T}_{\Sigma}$, denoted by $\operatorname{pos}(t)$, is defined by $\operatorname{pos}(t)=\{\epsilon\} \cup\left\{i p \mid i \in[k], p \in \operatorname{pos}\left(t_{i}\right)\right\}$ where $\sigma \in \Sigma^{(k)}$ and $t_{1}, \ldots, t_{k} \in \mathcal{T}_{\Sigma}$. The empty string $\epsilon$ is the position of the root of $t$, and the $i$ th child's position of $p \in \operatorname{pos}(t)$ is $p i$. We write $p \preceq p^{\prime}$ when $p$ is a prefix of $p^{\prime}$, that is, $p$ is an ancestor position of $p^{\prime}$, and $p \prec p^{\prime}$ when $p$ is a proper prefix of $p^{\prime}$. For $p, p^{\prime} \in \operatorname{pos}(t)$, let nca $\left(p, p^{\prime}\right)$ be the nearest common ancestor position of $p$ and $p^{\prime}$, that is, the longest common prefix of $p$ and $p^{\prime}$. For $p \in \operatorname{pos}(t),\left.t\right|_{p}$ denotes the subtree of $t$ at $p$, and $t\left[t^{\prime}\right]_{p}$ denotes the tree obtained from $t$ by replacing the subtree at $p$ with $t^{\prime}$. Let $\lambda_{t}(p)$ be the symbol of tree $t$ at $p$.

Let $X=\left\{x_{*}\right\} \cup\left\{x_{i} \mid i \geq 1\right\}$ be a set of variables of rank 0 , and for every $k \geq 1$, $X_{k}=\left\{x_{i} \mid i \in[k]\right\}$. For $V \subseteq X$, we often write $\mathcal{T}_{\Sigma}(V)$ to mean $\mathcal{T}_{\Sigma \cup V}$. A tree $t \in$ $\mathcal{T}_{\Sigma}(V)$ is linear if each variable in $V$ occurs at most once in $t$. Let $C_{\Sigma}(V)$ denote the set of linear trees in $T_{\Sigma}(V)$. Let $\overline{\mathcal{T}}_{\Sigma}(V)$ (resp. $\overline{\mathcal{C}}_{\Sigma}(V)$ ) be the set of trees in $\mathcal{T}_{\Sigma}(V)$ (resp. $\mathcal{C}_{\Sigma}(V)$ ) such that each variable in $V$ occurs at least once. Note that $\overline{\mathcal{T}}_{\Sigma \cup V}\left(V^{\prime}\right)$ denotes the set of trees in $\mathcal{T}_{\Sigma}\left(V \cup V^{\prime}\right)$ such that every variable in $V^{\prime}$ must occur at least once. For $t \in \mathcal{T}_{\Sigma}(X)$ and $\sigma \in \Sigma \cup X$, let $\operatorname{pos}_{\sigma}(t)$ be the set of the positions of $t$ at which $\sigma$ occurs, and $\operatorname{pos}_{Y}(t)=\bigcup_{\sigma \in Y} \operatorname{pos}_{\sigma}(t)$ for $Y \subseteq \Sigma \cup X$. Let $\operatorname{var}(t)$ be the set of variables of $t$, and $\operatorname{yield}_{X}: \mathcal{T}_{\Sigma}(X) \rightarrow X^{*}$ be the function such that yield $X_{X}(x)=x$ for every $x \in X$ and $\operatorname{yield}_{X}\left(\sigma\left(t_{1}, \ldots, t_{k}\right)\right)=\operatorname{yield}_{X}\left(t_{1}\right) \cdots \operatorname{yield}_{X}\left(t_{k}\right)$ for every $\sigma \in \Sigma^{(k)}$ and $t_{1}, \ldots, t_{k} \in \mathcal{T}_{\Sigma}(X)$. A tree $t \in \mathcal{T}_{\Sigma}(X)$ is normalized if yield $X_{X}(t)=x_{1} \cdots x_{k}$ for some $k \in \mathbb{N}$. Every mapping $\theta: V \rightarrow \mathcal{T}_{\Sigma}(X)$ with $V \subseteq X$ is called a substitution. It can be extended to $\theta: \mathcal{T}_{\Sigma}(V) \rightarrow \mathcal{T}_{\Sigma}(X)$ defined inductively as follows: $x \theta=\theta(x)$ for every $x \in V$ and $t \theta=\sigma\left(t_{1} \theta, \ldots, t_{k} \theta\right)$ for every $t=\sigma\left(t_{1}, \ldots, t_{k}\right) \in \mathcal{T}_{\Sigma}(V)$ where $\sigma \in \Sigma^{(k)}$. If $V=X_{k}$ and $x_{i} \theta=t_{i}$ for each $i \in[k]$, we also denote $t \theta$ by $t\left[t_{1}, \ldots, t_{k}\right]$, 
and if $V=\{x\}$ and $\theta(x)=t^{\prime}$, we denote $t \theta$ by $t\left[x \leftarrow t^{\prime}\right]$. In particular, if $V=\left\{x_{*}\right\}$ and $\theta\left(x_{*}\right)=t^{\prime}$, we denote $t \theta$ by $t\left[t^{\prime}\right]$ or often $t t^{\prime}$ without brackets.

A finite tree automaton (TA for short) is a 4-tuple $A=\left(Q, \Sigma, Q_{a}, \gamma\right)$, where $Q$ is a finite set of states, $\Sigma$ is an alphabet, $Q_{a} \subseteq Q$ is a set of accepting states, and $\gamma$ is a finite set of transition rules, each of which is of the form $\left(q, C\left[q_{1}, \ldots, q_{k}\right]\right)$ where $q, q_{1}, \ldots, q_{k} \in Q$ and $C \in \overline{\mathcal{C}}_{\Sigma}\left(X_{k}\right)$. The move relation $\Rightarrow_{A}$ of a TA $A=\left(Q, \Sigma, Q_{a}, \gamma\right)$ is defined as follows: if $\left(q, C\left[q_{1}, \ldots, q_{k}\right]\right) \in \gamma$ and $\left.t\right|_{p}=C\left[q_{1}, \ldots, q_{k}\right]$ where $p \in$ $\operatorname{pos}(t)$, then $t \Rightarrow_{A} t[q]_{p}$. The tree language recognized by $A$, denoted as $L(A)$, is $\left\{t \mid t \Rightarrow_{A}^{*} q_{a}, q_{a} \in Q_{a}\right\}$ where $\Rightarrow_{A}^{*}$ is the reflexive transitive closure of $\Rightarrow_{A}$. For a state $q$ of $A$, let $A(q)$ be a TA obtained from $A$ by replacing the set $Q_{a}$ of accepting states with the singleton $\{q\}$. A set $L$ of trees recognized by some TA is called a regular tree language, or we say $L$ is regular.

\subsection{Tree Transducers}

An extended bottom-up tree transducer (xbot) [9] is a 5-tuple $\left(Q, \Sigma, \Delta, Q_{f}, \delta\right)$, where $Q$ is a finite set of states, $\Sigma$ is an input alphabet, $\Delta$ is an output alphabet, $Q_{f} \subseteq Q$ is a set of final states, and $\delta$ is a set of transduction rules of the form $C_{l}\left[q_{1}\left(x_{1}\right), \ldots, q_{k}\left(x_{k}\right)\right] \rightarrow$ $q\left(t_{r}\right)$ where $k \in \mathbb{N}, C_{l} \in \overline{\mathcal{C}}_{\Sigma}\left(X_{k}\right), t_{r} \in \mathcal{T}_{\Delta}\left(X_{k}\right), q, q_{1}, \ldots, q_{k} \in Q$. A rule is normalized if its left-hand side is normalized. Without loss of generality, we can assume that every rule is normalized. A rule $\rho \in \delta$ is an $\epsilon$-rule if the left-hand side of $\rho$ is the form $q(x)$ where $q \in Q$ and $x \in X$, and it is input-consuming otherwise. Let $T=\left(Q, \Sigma, \Delta, Q_{f}, \delta\right)$ be an xbot. $T$ is a bottom-up tree transducer (bot) if the lefthand side of every rule in $\delta$ contains exactly one symbol in $\Sigma$. Also, we denote by an $\operatorname{xbot}^{-e}$ an xbot without $\epsilon$-rules. $T$ is a linear extended bottom-up tree transducer ( $l$-xbot) if the tree $t_{r}$ in the right-hand side of each rule in $\delta$ is linear.

The move relation $\Rightarrow_{T}$ of an xbot $T=\left(Q, \Sigma, \Delta, Q_{f}, \delta\right)$ is defined as follows: $t \Rightarrow_{T}^{\rho} t^{\prime}$ for a rule $\rho=\left(C_{l}\left[q_{1}\left(x_{1}\right), \ldots, q_{k}\left(x_{k}\right)\right] \rightarrow q\left(t_{r}\right)\right) \in \delta$ if there exists a position $p \in \operatorname{pos}(t)$ such that $\left.t\right|_{p}=C_{l}\left[q_{1}\left(t_{1}\right), \ldots, q_{k}\left(t_{k}\right)\right]$ where $t_{1}, \ldots, t_{k} \in \mathcal{T}_{\Delta}(X)$ and $t^{\prime}=t\left[q\left(t_{r}\left[t_{1}, \ldots, t_{k}\right]\right)\right]_{p}$, and $t \Rightarrow_{T} t^{\prime}$ if there exists $\rho \in \delta$ such that $t \Rightarrow_{T}^{\rho} t^{\prime}$. The transformation induced by $T$, denoted as $\llbracket T \rrbracket$, is the relation defined as $\left\{\left(t, t^{\prime}\right) \mid t \Rightarrow_{T}^{*}\right.$ $\left.q_{f}\left(t^{\prime}\right), t \in \mathcal{T}_{\Sigma}, t^{\prime} \in \mathcal{T}_{\Delta}, q_{f} \in Q_{f}\right\}$ where $\Rightarrow_{T}^{*}$ is the reflexive transitive closure of $\Rightarrow_{T}$. The domain of $T$, denoted by $\operatorname{dom}(T)$, is $\left\{t \mid\left(t, t^{\prime}\right) \in \llbracket T \rrbracket\right\}$, and the range of $T$, denoted by $\operatorname{rng}(T)$, is $\left\{t^{\prime} \mid\left(t, t^{\prime}\right) \in \llbracket T \rrbracket\right\}$. For a tree $t, \llbracket T \rrbracket(t)=\left\{t^{\prime} \mid\left(t, t^{\prime}\right) \in \llbracket T \rrbracket\right\}$. For a TA $A$, the image $T(A)$ of $L(A)$ by $T$ is $\left\{t^{\prime} \mid\left(t, t^{\prime}\right) \in \llbracket T \rrbracket, t \in L(A)\right\}$. For a state $q$ of $T$, let $T(q)$ be an xbot obtained from $T$ by replacing the set $Q_{f}$ of final states with the singleton $\{q\}$.

The tree transducers $T$ and $T^{\prime}$ are equivalent if $\llbracket T \rrbracket=\llbracket T^{\prime} \rrbracket$. For tree transducers $T_{1}$ and $T_{2}, \llbracket T_{2} \rrbracket \circ \llbracket T_{1} \rrbracket=\left\{\left(t, t^{\prime}\right) \mid\left(t, t^{\prime \prime}\right) \in \llbracket T_{1} \rrbracket,\left(t^{\prime \prime}, t^{\prime}\right) \in \llbracket T_{2} \rrbracket\right\}$. A transducer $T$ is said to be single-valued (or functional) if any two pairs of $\left(t, t^{\prime}\right)$ and $\left(t, t^{\prime \prime}\right)$ in $\llbracket T \rrbracket$ satisfy $t^{\prime}=t^{\prime \prime}$. We denote the unique output tree of $T$ on a tree $t$ by $T(t)$. It is known that the single-valuedness of bots is decidable in polynomial time [10]. We use the prefix ' $s$ ' to represent that a transducer is single-valued, e.g., we write for short an $s$-xbot to denote a single-valued xbot.

Without loss of generality, we assume that any alphabet contains a special symbol $\perp$, which means "no output" and does not occur in any final output tree. We recall the 
notion of reducedness [10], which is defined for bots but can be naturally applied to xbots. An xbot $T=\left(Q, \Sigma, \Delta, Q_{f}, \delta\right)$ is called reduced if and only if the following two conditions hold:

1. $T$ has no useless states, that is, for every state $q \in Q$, there exists a tree $t=C t_{s} \in$ $\operatorname{dom}(T)$ where $C \in \overline{\mathcal{C}}_{\Sigma}\left(\left\{x_{*}\right\}\right)$ such that $t \Rightarrow_{T}^{*} C\left[q\left(t_{s}^{\prime}\right)\right] \Rightarrow_{T}^{*} q_{f}\left(t^{\prime}\right)$ for some $q_{f} \in Q_{f}$ and $t_{s}^{\prime}, t^{\prime} \in \mathcal{T}_{\Delta}$.

2. There exists a subset $U(T)$ of $Q$ such that for every rule $C_{l}\left[q_{1}\left(x_{1}\right), \ldots\right.$, $\left.q_{k}\left(x_{k}\right)\right] \rightarrow q\left(t_{r}\right) \in \delta$,

- if $q \in U(T)$ then $t_{r}=\perp$ and $q_{i} \in U(T)$ for each $i \in[k]$, and

- if $q \notin U(T)$ then (1) $t_{r} \neq \perp$ and (2) for each $i \in[k], q_{i} \in U(T)$ if and only if $x_{i} \notin \operatorname{var}\left(t_{r}\right)$.

3. If $q \in Q_{f}$ then $q$ does not occur in the left-hand side of any rule in $\delta$.

Note that for any $q \in U(T)$ and $t=C t_{s} \in \operatorname{dom}(T)$ where $C \in \overline{\mathcal{C}}_{\Sigma}\left(\left\{x_{*}\right\}\right)$, if $t \Rightarrow_{T}^{*} C\left[q\left(t_{2}^{\prime}\right)\right]$ then $t_{2}^{\prime}=\perp$ and the final output for $t$ does not contain $\perp$. That is, the intermediate output at $q$ is always $\perp$ and it is eventually abandoned. Conversely, for $q \in Q-U(T)$, the intermediate output at $q$ is in $\mathcal{T}_{\Delta-\{\perp\}}$ and it is contained in the final output. For every xbot $T$, a reduced xbot equivalent with $T$ can be constructed in linear time in the same way as the construction for bots [10] (see also Appendix A).

\subsection{Determinacy and Subsumption of Tree Transducers}

Let $\Pi_{1}$ and $\Pi_{2}$ be arbitrary classes of tree transducers.

Definition 1 (Determinacy). Let $T_{1}$ and $T_{2}$ be tree transducers in $\Pi_{1}$ and $\Pi_{2}$, respectively, such that $\operatorname{dom}\left(T_{2}\right) \subseteq \operatorname{dom}\left(T_{1}\right) . T_{1}$ determines $T_{2}$ iff there exists a partial function $f$ such that $\llbracket T_{2} \rrbracket=f \circ \llbracket T_{1} \rrbracket . \Pi_{1}$ is called the determiner class and $\Pi_{2}$ is called the determinee class.

Definition 2 (Subsumption). Let $T_{1}$ and $T_{2}$ be tree transducers in $\Pi_{1}$ and $\Pi_{2}$, respectively, such that $\operatorname{dom}\left(T_{2}\right) \subseteq \operatorname{dom}\left(T_{1}\right) . T_{1}$ subsumes $T_{2}$ with respect to $\Pi_{2}$ iff there exists a single-valued transducer $T_{3} \in \Pi_{2}$ such that $\llbracket T_{2} \rrbracket=\llbracket T_{3} \rrbracket \circ \llbracket T_{1} \rrbracket$.

From the definition, if $T_{1}$ subsumes $T_{2}$ then $T_{1}$ determines $T_{2}$. Conversely, even if there exists some function $f$ such that $\llbracket T_{2} \rrbracket=f \circ \llbracket T_{1} \rrbracket, f$ cannot always be induced by some transducer in $\Pi_{2}$ in general.

If determinacy is decidable for a determiner class $\Pi_{1}$ and a determinee class $\Pi_{2}$, we simply say determinacy is decidable for $\left(\Pi_{1}, \Pi_{2}\right)$. We will use a similar notation for subsumption.

\section{Determinacy}

\subsection{Decidability for ( $s l$-xbots, $s$-bots)}

We consider the problem of deciding whether, given single-valued linear xbot ( $s l$-xbot) $T_{1}$ and single-valued bot ( $s$-bot) $T_{2}$ such that $\operatorname{dom}\left(T_{2}\right) \subseteq \operatorname{dom}\left(T_{1}\right), T_{1}$ determines $T_{2}$ or not. Our approach is based on the following proposition. 


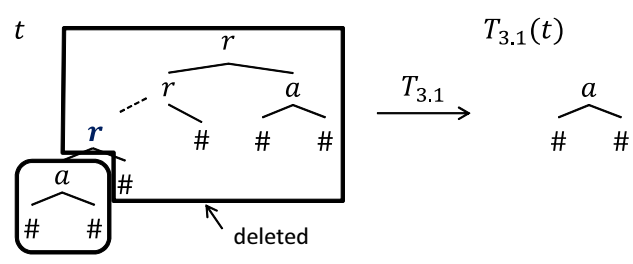

Fig. 2. A transducer $T_{3.1}$

Proposition 1. For any single-valued transducers $T_{1}$ and $T_{2}$ such that $\operatorname{dom}\left(T_{2}\right) \subseteq$ $\operatorname{dom}\left(T_{1}\right), T_{1}$ determines $T_{2}$ if and only if $\llbracket T_{2} \rrbracket \circ \llbracket T_{1} \rrbracket^{-1}$ is a partial function, where $\llbracket T_{1} \rrbracket^{-1}=\left\{\left(t^{\prime}, t\right) \mid\left(t, t^{\prime}\right) \in \llbracket T_{1} \rrbracket\right\}$.

According to Proposition 1, given $s l$-xbot $T_{1}$ and $s$-bot $T_{2}$, our decision algorithm works as follows:

Step 1: Construct a transducer $T_{1}^{\text {inv }}$ such that $\llbracket T_{1}^{i n v} \rrbracket=\llbracket T_{1} \rrbracket^{-1}$;

Step 2: Construct a transducer $T_{3}$ such that $\llbracket T_{3} \rrbracket=\llbracket T_{2} \rrbracket \circ \llbracket T_{1}^{\text {inv }} \rrbracket$;

Step 3: Decide whether $T_{3}$ is single-valued.

In Step 1, the inverse transducer $T_{1}^{i n v}$ of $T_{1}$ is computed. $T_{1}^{i n v}$ is not necessarily an $l$-xbot. Due to this, we introduce a slightly larger class, linear extended bottom-up tree transducers with grafting $\left(l-\mathrm{xbot}^{+\mathrm{g}}\right.$ for short), that can represent not only inverses of $l$-xbots but also the composition of the inverses with $s$-bots. In Step 2, an $\operatorname{xbot}^{+\mathrm{g}} T_{3}$ which represents the composition of $T_{1}^{i n v}$ followed by $T_{2}$ is constructed. Lastly, it is determined whether the composition transducer $T_{3}$ is single-valued.

Before we explain the detail of each step, we give an example, which shows that even the inverse of an $s l$-bot cannot always be expressed by any $l$-xbot.

Example 1. Let $\Sigma=\{r, a, \#\}$ and $\Delta=\{a, \#\}$. Consider an sl-bot $T_{3.1}=$ $\left(\left\{q_{r}, q\right\}, \Sigma, \Delta,\left\{q_{r}\right\}, \delta\right)$ where

$$
\begin{aligned}
& \delta=\left\{\# \rightarrow q(\#), \quad a\left(q\left(x_{1}\right), q\left(x_{2}\right)\right) \rightarrow q\left(a\left(x_{1}, x_{2}\right)\right),\right. \\
& \left.r\left(q\left(x_{1}\right), q\left(x_{2}\right)\right) \rightarrow q_{r}\left(x_{1}\right), \quad r\left(q_{r}\left(x_{1}\right), q\left(x_{2}\right)\right) \rightarrow q_{r}\left(x_{1}\right)\right\} .
\end{aligned}
$$

In Fig. 2, $t$ is transformed by $T_{3.1}$, which leaves only the subtree at the left child of the bottom-most $r$-node. There is an infinite number of trees $t^{\prime}$ such that $T_{3.1}\left(t^{\prime}\right)=T_{3.1}(t)$ because the inverse of $T_{3.1}$ allows to insert any number of $r$-labeled ancestor nodes having arbitrary trees in $\mathcal{T}_{\Sigma-\{r\}}$ as their right subtrees. For any $l$-xbot $T$ without $\epsilon$ rules, the image of a tree $t$ by $T$ is finite. Even if $\epsilon$-rules are allowed, no $l$-xbot allows to insert a node having an arbitrary tree in $\mathcal{T}_{\Sigma-\{r\}}$ as its right subtree. Therefore, there is no $l$-xbot $T$ such that $\llbracket T \rrbracket=\llbracket T_{3.1} \rrbracket^{-1}$.

To express the inverse of $T_{3.1}$ in Example 1, a transducer has to, for an input tree, insert any number of internal nodes and subtrees non-deterministically. To capture the inverse of $s l$-xbots, we extend xbots by grafting. We denote a tree transducer in the class 
by an $\mathrm{xbot}^{+\mathrm{g}}$ for short. A grafting is represented by a special variable $\langle L\rangle$, called a $\mathrm{g}$ variable, where $L \subseteq \mathcal{T}_{\Delta}$. When $L=L(A)$ where $A$ is a TA over $\Delta$, we often write $\langle A\rangle$ instead of $\langle L(A)\rangle$. A g-variable can occur as a symbol of rank 0 in the right-hand side of a rule. Let $G(\Delta)$ be the set of all the g-variables $\langle L\rangle$ where $L \subseteq \mathcal{T}_{\Delta}$. Let $\tilde{\mathcal{T}}_{\Delta}\left(X_{i}\right)$ denote the set of trees over $\Delta$ with $X_{i}$ and $G(\Delta)$. Note that for $\tilde{t} \in \tilde{\mathcal{T}}_{\Delta}\left(X_{i}\right), \operatorname{var}(\tilde{t})$ does not contain any g-variable. For $\tilde{t} \in \tilde{\mathcal{T}}_{\Delta}\left(X_{i}\right)$, let $S(\tilde{t})$ be the set of trees in $\mathcal{T}_{\Delta}\left(X_{i}\right)$ obtained from $\tilde{t}$ by replacing each g-variable $\langle L\rangle$ with a tree in $L$.

Formally, a transduction rule of an $\mathrm{xbot}^{+\mathrm{g}}$ is the form $C_{l}\left[q_{1}\left(x_{1}\right), \ldots, q_{k}\left(x_{k}\right)\right] \rightarrow$ $q\left(\tilde{t}_{r}\right)$ where $k \in \mathbb{N}, C_{l} \in \overline{\mathcal{C}}_{\Sigma}\left(X_{k}\right), \tilde{t}_{r} \in \tilde{\mathcal{T}}_{\Delta}\left(X_{k}\right)$, and $q, q_{1}, \ldots, q_{k}$ are states. The move relation by a rule $C_{l}\left[q_{1}\left(x_{1}\right), \ldots, q_{k}\left(x_{k}\right)\right] \rightarrow q\left(\tilde{t}_{r}\right)$ is as follows: if $t_{p}=$ $C_{l}\left[q_{1}\left(t_{1}\right), \ldots, q_{k}\left(t_{k}\right)\right]$ where $t_{1}, \ldots, t_{k} \in \mathcal{T}_{\Delta}$, then $t \Rightarrow t\left[q\left(t_{r}\left[t_{1}, \ldots, t_{k}\right]\right)\right]_{p}$ where $t_{r} \in S\left(\tilde{t}_{r}\right)$.

For an $\mathrm{xbot}^{+\mathrm{g}}$, we write an $\mathrm{xbot}^{+\mathrm{g}(\mathrm{R})}$ when $L$ is regular for each g-variable $\langle L\rangle$. Also, we write an $\mathrm{xbot}^{+\mathrm{g}(\mathrm{B}(\mathrm{R}))}$ when each $\mathrm{g}$-variable is in the form of $\langle T(A)\rangle$ for some bot $T$ and TA $A$.

Example 2. Consider an $l$-xbot ${ }^{+\mathrm{g}(\mathrm{R})} T_{3.2}=\left(\left\{q, q_{r}\right\}, \Delta, \Sigma,\left\{q_{r}\right\}, \delta^{\prime}\right)$ where

$$
\begin{aligned}
\delta^{\prime}=\left\{\# \rightarrow q(\#), \quad a\left(q\left(x_{1}\right), q\left(x_{2}\right)\right) \rightarrow q\left(a\left(x_{1}, x_{2}\right)\right),\right. & \\
& \left.q\left(x_{1}\right) \rightarrow q_{r}\left(r\left(x_{1},\langle A\rangle\right)\right), \quad q_{r}\left(x_{1}\right) \rightarrow q_{r}\left(r\left(x_{1},\langle A\rangle\right)\right)\right\}
\end{aligned}
$$

and $A$ is a TA such that $L(A)=\mathcal{T}_{\Sigma-\{r\}}$. Then, $T_{3.2}$ induces the inverse of $T_{3.1}$.

Steps 1 to 3 of the decision algorithm can be refined as follows.

Step 1: Inversion of $s \boldsymbol{l}$-xbots. We provide a way to construct an $l$-xbot ${ }^{+g}$ representing the inverse of an $s l$-xbot. Intuitively, we just swap the input and output of each rules. However, we must take care of variables occurring only in the left-hand side, which mean deletions of subtrees. In swapping, g-variables are added instead of the variables.

Let $T=\left(Q, \Sigma, \Delta, Q_{f}, \delta\right)$ be an $l$-xbot. The swapping procedure is as follows.

1. Construct a TA $A_{T}=\left(Q, \Sigma, Q_{f}, \gamma\right)$ where $\gamma=\left\{\left(q, C_{l}\left[q_{1}, \ldots, q_{k}\right]\right)\right.$ $\left.C_{l}\left[q_{1}\left(x_{1}\right), \ldots, q_{k}\left(x_{k}\right)\right] \rightarrow q\left(C_{r}\right) \in \delta\right\}$. Note that $A_{T}$ recognizes $\operatorname{dom}(T)$.

2. Construct an $l$-xbot ${ }^{+\mathrm{g}(\mathrm{R})} T^{\prime}=\left(Q, \Delta, \Sigma, Q_{f}, \delta^{\prime}\right)$ such that $\delta^{\prime}$ is the smallest set satisfying the following condition: Let $C_{l}\left[q_{1}\left(x_{1}\right), \ldots, q_{k}\left(x_{k}\right)\right] \rightarrow q\left(C_{r}\right)$ be an arbitrary rule in $\delta$. Let $\theta_{l}$ be the substitution such that $\theta_{l}\left(x_{i}\right)=q_{i}\left(x_{i}\right)$ for each $i \in[k]$, $\theta_{r}$ be the substitution such that $\theta_{r}\left(x_{i}\right)=x_{i}$ if $x_{i} \in \operatorname{var}\left(C_{r}\right)$ and $\theta_{r}\left(x_{i}\right)=\left\langle A_{T}\left(q_{i}\right)\right\rangle$ otherwise. Moreover, let $\theta_{n}$ be the substitution for normalization, which is the bijective function from $\operatorname{var}\left(C_{r}\right)$ to $X_{k^{\prime}}\left(k^{\prime}=\left|\operatorname{var}\left(C_{r}\right)\right|\right)$ making $\left(C_{r} \theta_{l}\right) \theta_{n}$ normalized. Then, $\left(C_{r} \theta_{l}\right) \theta_{n} \rightarrow\left(C_{l} \theta_{r}\right) \theta_{n} \in \delta^{\prime}$.

Lemma 1. For any $l$-xbot $T$, an $l$-xbot ${ }^{+\mathrm{g}(\mathrm{R})} T^{i n v}$ such that $\llbracket T^{i n v} \rrbracket=\llbracket T \rrbracket^{-1}$ can be constructed.

Proof. It can be shown by induction on move relations of the transducers that the inverse transducer $T^{i n v}$ of $T$ is correctly constructed by the swapping. 
Step 2: Composition of $l$-xbot ${ }^{+\mathrm{g}(\mathrm{R})}$ and $s$-bot. This step constructs an $\mathrm{xbot}^{+\mathrm{g}}$ equivalent with the composition of the $l$-xbot ${ }^{+\mathrm{g}(\mathrm{R})} T_{1}^{i n v}$ followed by an $s$-bot $T_{2}$.

Lemma 2. For any $l$-xbot ${ }^{+\mathrm{g}(\mathrm{R})} T$ and bot $T^{\prime}$, an $x b o t^{+\mathrm{g}(\mathrm{B}(\mathrm{R}))} T^{\prime \prime}$ such that $\llbracket T^{\prime \prime} \rrbracket=$ $\llbracket T^{\prime} \rrbracket \circ \llbracket T \rrbracket$ can be constructed.

Proof. The lemma can be shown in a similar way to the proof of the closure property of $l$-bots under the composition [7,11]. The difference is the existence of g-variables. Recall that a tree $t$ in $L(A)$ is inserted at g-variable $\langle A\rangle$. On the composition transducer, we just insert the image of $t$ by $T^{\prime}(q)$ where $q$ is the state at which $T^{\prime}$ processes $t$ in the tree output by $T$. That is, we replace $\langle A\rangle$ with $\left\langle T^{\prime}(q)(A)\right\rangle$.

Step 3: Deciding single-valuedness of $\mathbf{x b o t}^{+\mathrm{g}(\mathbf{B}(\mathbf{R}))}$. This step decides whether the xbot $^{+g(B(R))}$ obtained in Step 2 is single-valued. It is known that single-valuedness of bots is decidable in polynomial time [10]. However, the class of transformations induced by $\mathrm{xbot}^{+\mathrm{g}_{\mathrm{S}}}$ is a proper superclass of the class induced by bots.

Let $T_{3}$ be the $\operatorname{xbot}^{+\mathrm{g}(\mathrm{B}(\mathrm{R}))}$ obtained in Step 2. The overview of Step 3 is as follows:

Step 3-1 Construct a reduced xbot $T_{3.1}$ equivalent with $T_{3}$ by eliminating g-variables. If there is no xbot equivalent with $T_{3}$, answer that $T_{3}$ is not single-valued and halt. Otherwise, go to 3-2.

Step 3-2 Construct a reduced $\operatorname{xbot}^{-e} T_{3.2}$ equivalent with $T_{3.1}$. If there is no $\mathrm{xbot}^{-e}$ equivalent with $T_{3.1}$, answer that $T_{3}$ is not single-valued and halt. Otherwise, go to 3-3.

Step 3-3 Decide whether $T_{3.2}$ is single-valued or not.

We further refine the above sub-steps as follows.

Step 3-1: Eliminating g-variables. We show the following lemma for Step 3-1.

Lemma 3. Let $T=\left(Q, \Sigma, \Delta, Q_{f}, \delta\right)$ be a reduced $x b o t^{+\mathrm{g}}$. If $T$ has a rule whose righthand side has a state $q \in Q-U(T)$ and a g-variable $\langle L\rangle$ such that $|L| \geq 2$, then $T$ is not single-valued.

Proof. Assume that $T$ has a rule $C_{l}\left[q_{1}\left(x_{1}\right), \ldots, q_{k}\left(x_{k}\right)\right] \rightarrow q\left(\tilde{t}_{r}\right)$ where $q \in Q-U(T)$ and $\tilde{t}_{r}$ has a g-variable $\langle L\rangle$ such that $|L| \geq 2$. Since $T$ is reduced, there exist $t=$ $C C_{l}\left[t_{1}, \ldots, t_{k}\right] \in \operatorname{dom}(T)$ where $C \in \overline{\mathcal{C}}_{\Sigma}\left(\left\{x_{*}\right\}\right), t^{\prime} \in \overline{\mathcal{T}}_{\Delta}\left(\left\{x_{*}\right\}\right), t_{1}^{\prime}, \ldots, t_{k}^{\prime} \in \mathcal{T}_{\Delta}$, and $q_{f} \in Q_{f}$ such that $t \Rightarrow_{T}^{*} C C_{l}\left[q_{1}\left(t_{1}^{\prime}\right), \ldots, q_{k}\left(t_{k}^{\prime}\right)\right] \Rightarrow_{T} C\left[q\left(t_{r}\left[t_{1}^{\prime}, \ldots, t_{k}^{\prime}\right]\right)\right] \Rightarrow_{T}^{*}$ $q_{f}\left(t^{\prime} t_{r}\left[t_{1}^{\prime}, \ldots, t_{k}^{\prime}\right]\right)$ for any $t_{r} \in S\left(\tilde{t}_{r}\right)$. Since $|L| \geq 2, S\left(\tilde{t}_{r}\right)$ has at least two distinct trees $t_{r}^{1}$ and $t_{r}^{2}$. Also, the positions of each variable of $t_{r}^{1}$ and $t_{r}^{2}$ are identical. Hence, $t_{r}^{1}\left[t_{1}^{\prime}, \ldots, t_{k}^{\prime}\right] \neq t_{r}^{2}\left[t_{1}^{\prime}, \ldots, t_{k}^{\prime}\right]$ and thus the final outputs $t^{\prime} t_{r}^{1}\left[t_{1}^{\prime}, \ldots, t_{k}^{\prime}\right]$ and $t^{\prime} t_{r}^{2}\left[t_{1}^{\prime}, \ldots, t_{k}^{\prime}\right]$ are different. Therefore, $|\llbracket T \rrbracket(t)| \geq 2$.

For a bot $T$, a TA $A$ and any $k \in \mathbb{N}$, it can be checked whether $|T(A)| \geq k$. From the above lemma, Step 3-1 can be done as follows:

(i) For each rule with grafting $\langle T(A)\rangle$ of $T_{3}$,

- if $T(A)=\emptyset$ then delete the rule, and 
- if $T(A)=\{t\}$ for some tree $t$ then replace $\langle T(A)\rangle$ with $t$.

(ii) Construct an equivalent reduced $\mathrm{xbot}^{+\mathrm{g}(\mathrm{B}(\mathrm{R}))} T_{3.1}$.

(iii) If $T_{3.1}$ has a rule containing $\langle T(A)\rangle$ with $|T(A)| \geq 2$, answer that $T_{3}$ is not singlevalued and halt.

If the condition at (iii) does not hold, $T_{3.1}$ has no g-variable. Thus, $T_{3.1}$ is an xbot.

Step 3-2: Eliminating $\epsilon$-rules. We show two lemmas before giving the procedure of Step 3-2. We will use an idea similar to the proof of Proposition 10 of [9].

We say that a nonempty subset $\delta_{e}$ of $\epsilon$-rules is repeatedly-producing at state $q$ if $q\left(x_{*}\right) \Rightarrow_{\delta_{e}}^{*} q(t)$ for some tree $t \in \overline{\mathcal{T}}_{\Delta}\left(\left\{x_{*}\right\}\right)-\left\{x_{*}\right\}$, where $\Rightarrow_{\delta_{e}}^{*}$ means zero ore more applications of rules in $\delta_{e}$.

Lemma 4. Let $T=\left(Q, \Sigma, \Delta, Q_{f}, \delta\right)$ be a reduced xbot. If there is a subset $\delta_{e}$ of $\epsilon$ rules in $\delta$ repeatedly-producing at some $q \in Q-U(T)$, then $T$ is not single-valued.

Proof. Assume that there is a subset $\delta_{e}$ of $\epsilon$-rules in $\delta$ repeatedly-producing at some $q \in$ $Q-U(T)$. Then, there are trees $t \in \mathcal{T}_{\Sigma}, t^{\prime} \in \mathcal{T}_{\Delta}, D \in \overline{\mathcal{C}}_{\Sigma}\left(\left\{x_{*}\right\}\right), t_{c}, t_{D} \in \overline{\mathcal{T}}_{\Delta}\left(\left\{x_{*}\right\}\right)$, and $q_{f} \in Q_{f}$ such that $t_{c}$ has at least one symbol in $\Delta$ and $D[t] \Rightarrow_{T}^{*} D\left[q\left(t^{\prime}\right)\right] \Rightarrow_{T}^{*}$ $D\left[q\left(t_{c} t^{\prime}\right)\right] \Rightarrow_{T}^{*} D\left[q\left(t_{c}^{n} t^{\prime}\right)\right] \Rightarrow_{T}^{*} q_{f}\left(t_{D} t_{c}^{n} t^{\prime}\right)$ for any positive integer $n$.

After the fashion of the reference [9], we call a state $q \in Q$ an end state if there exists an input-consuming rule whose left-hand side has $q$. The set of all end states of $Q$ is denoted by $E(T)$. For each input-consuming rule $\rho=\left(C_{l}\left[q_{1}\left(x_{1}\right), \ldots, q_{k}\left(x_{k}\right)\right] \rightarrow\right.$ $\left.q\left(t_{r}\right)\right) \in \delta$, let $\operatorname{rhs}(\rho)=\left\{q^{\prime}(t) \mid q\left(t_{r}\right) \Rightarrow_{T}^{*} q^{\prime}(t), q^{\prime} \in E(T) \cup Q_{f}\right\}$. Note that only $\epsilon$-rules can be used in the derivation $q\left(t_{r}\right) \Rightarrow_{T}^{*} q^{\prime}(t)$.

Lemma 5. Let $T=\left(Q, \Sigma, \Delta, Q_{f}, \delta\right)$ be a reduced xbot. If there is no subset $\delta_{e}$ of $\epsilon$-rules in $\delta$ repeatedly-producing at any $q \in Q-U(T)$, then $\operatorname{rhs}(\rho)$ is finite for every rule $\rho$ of $T$ and an $x^{-e}{ }^{-e}$ equivalent with $T$ can be constructed.

Proof. Assume that there is no subset $\delta_{e}$ of $\epsilon$-rules in $\delta$ repeatedly-producing at any $q \in Q-U(T)$. By the assumption, for any tree $t, q(t) \Rightarrow_{T}^{+} q\left(t^{\prime}\right)$ and $t \neq t^{\prime}$ imply that $t^{\prime}$ does not contain any variable. That is, $q(t) \Rightarrow_{T}^{+} q\left(t^{\prime}\right) \Rightarrow_{T}^{*} q\left(t^{\prime \prime}\right)$ and $t \neq t^{\prime}$ imply $t^{\prime}=t^{\prime \prime}$. Thus, suppose that $n_{r}$ is the number of rules of $T$, and then for each rule $\rho=\left(C_{l}\left[q_{1}\left(x_{1}\right), \ldots, q_{k}\left(x_{k}\right)\right] \rightarrow q\left(t_{r}\right)\right) \in \delta, \operatorname{rhs}(\rho)=\left\{q^{\prime}(t) \mid q\left(t_{r}\right) \Rightarrow_{T}^{i} q^{\prime}(t), q^{\prime} \in\right.$ $\left.E(T) \cup Q_{f}, i \in\left[n_{r}\right]\right\}$ where $\Rightarrow_{T}^{i}$ means the move relation by $i$ times applications of rules. Therefore, $\operatorname{rhs}(\rho)$ is finite. Then, we can construct an equivalent $\operatorname{xbot}^{-e} T_{e}=$ $\left(Q, \Sigma, \Delta, Q_{f}, \delta^{\prime}\right)$ where $\delta^{\prime}=\bigcup_{l \rightarrow r \in \delta^{\Sigma}}\left\{l \rightarrow r^{\prime} \mid r^{\prime} \in \operatorname{rhs}(l \rightarrow r)\right\}$ and $\delta^{\Sigma}$ is the subset of input-consuming rules of $\delta$.

According to Lemmas 4 and 5, Step 3-2 consists of the following two substeps:

(i) Construct the weighted graph $G_{r p}=\left(Q-U\left(T_{3.1}\right), E_{e}\right)$ from $T_{3.1}=$ $\left(Q, \Sigma, \Delta, Q_{f}, \delta\right)$ where $E_{e}=\left\{\left(q, q^{\prime}\right) \mid q\left(x_{1}\right) \rightarrow q^{\prime}(t) \in \delta, t \in \overline{\mathcal{T}}_{\Delta}\left(\left\{x_{1}\right\}\right)\right\}$, and the weight of each $\left(q, q^{\prime}\right)$ is 1 if there is a rule $q\left(x_{1}\right) \rightarrow q^{\prime}(t)$ such that $t$ includes at least one output symbol, and otherwise 0 . Find a cycle whose weight is at least one. If such a cycle exists, answer that $T_{3}$ is not single-valued and halt.

(ii) Construct an equivalent reduced $\operatorname{xbot}^{-e} T_{3.2}$. 
Step 3-3: Deciding single-valuedness of $x$ bot $^{-e}$. In this substep, it is decided whether $T_{3.2}$ is single-valued or not. The idea of the proof is the same as that of the proof of the decidability of $k$-valuedness of bottom-up tree transducers [12]. While the proof in [12] uses the Engelfriet's property, we use a variant of the property (Lemma 6) to prove the decidability of single-valuedness of $\mathrm{xbot}^{-e}$ S.

We give some notations for the property. Let $\mathcal{T}_{\Sigma}\left[X_{n}\right]=\overline{\mathcal{T}}_{\Sigma}\left(X_{n}\right) \cup \mathcal{T}_{\Sigma}$, that is, every $t \in \mathcal{T}_{\Sigma}\left[X_{n}\right]$ has all the variables in $X_{n}$ or has no variable. For $t, s \in \mathcal{T}_{\Sigma}\left[X_{n}\right], t s$ is the tree obtained from $t$ by replacing each variable with $s$. Note that $t s=t$ if $t$ has no variable. For $m \in[n]$, let $\mathcal{T}_{\Sigma}^{n, m}\left[X_{n}\right]=\mathcal{T}_{\Sigma}^{m-1} \times \mathcal{T}_{\Sigma}\left[X_{n}\right] \times \mathcal{T}_{\Sigma}^{n-m}$. For $\mathbf{t} \in \mathcal{T}_{\Sigma}^{n, m}\left[X_{n}\right]$, we denote by $t^{(i)}$ the $i$ th element of $\mathbf{t}$, i.e., $\mathbf{t}=\left(t^{(1)}, \ldots, t^{(n)}\right)$. For $s \in \mathcal{T}_{\Sigma}\left[X_{n}\right]$ and $\mathbf{t} \in \mathcal{T}_{\Sigma}^{n, m}\left[X_{n}\right], s \mathbf{t}$ is the tree obtained from $s$ by replacing $x_{i}$ with $t^{(i)}$ for all $i \in[n]$. Let $\mathbf{t u}=\left(t^{(1)} \mathbf{u}, \ldots, t^{(n)} \mathbf{u}\right)$ for $\mathbf{u} \in \mathcal{T}_{\Sigma}^{n, m}\left[X_{n}\right]$. Notice that since $\mathbf{t} \in \mathcal{T}_{\Sigma}^{n, m}\left[X_{n}\right]$, so is tu. For $\mathbf{t}_{1}, \mathbf{t}_{2}, \mathbf{t}_{3}, \mathbf{t}_{4}, \mathbf{t}_{5} \in \mathcal{T}_{\Sigma}^{n, m}\left[X_{n}\right]$ and $S=\left\{i_{1}, \ldots, i_{|S|}\right\} \subseteq[1,5]$, let $\mathbf{t}_{S}=\mathbf{t}_{i_{1}} \cdots \mathbf{t}_{i_{|S|}}$ where $i_{j}<i_{j+1}$ for $j \in[|S|-1]$.

Now, we give a variant of Engelfriet's Property (see Appendix B.1).

Lemma 6. Let $n, n^{\prime}$ be arbitrary positive integers, and $m \in[n], m^{\prime} \in\left[n^{\prime}\right]$. Suppose that $t_{0} \in \mathcal{T}_{\Sigma}\left[X_{n}\right], \mathbf{t}_{1}, \mathbf{t}_{2}, \mathbf{t}_{3}, \mathbf{t}_{4}, \mathbf{t}_{5} \in \mathcal{T}_{\Sigma}^{n, m}\left[X_{n}\right], t_{0}^{\prime} \in \mathcal{T}_{\Sigma}\left[X_{n^{\prime}}\right], \mathbf{t}_{1}^{\prime}, \mathbf{t}_{2}^{\prime}, \mathbf{t}_{3}^{\prime}, \mathbf{t}_{4}^{\prime}, \mathbf{t}_{5}^{\prime} \in$ $\mathcal{T}_{\Sigma}^{n^{\prime}, m^{\prime}}\left[X_{n^{\prime}}\right]$. If $t_{0} \mathbf{t}_{S}=t_{0}^{\prime} \mathbf{t}_{S}^{\prime}$ for every $S$ such that $\{5\} \subseteq S \subset[1,5]$, then $t_{0} \mathbf{t}_{[1,5]}=$ $t_{0}^{\prime} \mathbf{t}_{[1,5]}^{\prime}$.

Next, in order to argue in a similar way to the proof of Theorem 2.2(i) in the reference [12], we decompose the left-hand side of each rule into several rules each of which has only one input symbol. Actually, we construct a multi bottom-up tree transducer (mbot) [9] equivalent with a given $\mathrm{xbot}^{-e}$. An mbot is a bot whose states might have ranks different from one. Intuitively, we decompose each rule $\rho$ of the $\mathrm{xbot}^{-e}$ by adding a state for each intermediate position of the left-hand side tree $l$ of $\rho$. The added states might have rank different from one to maintain two or more intermediate output trees until the obtained mbot reaches the state corresponding to the root of $l$ (see Appendix B.2).

Example 3. Assume that an $\mathrm{xbot}^{-e} T$ has the transduction rule $\rho=$ $a\left(b\left(q_{1}\left(x_{1}\right), q_{2}\left(x_{2}\right), q_{3}\left(x_{3}\right)\right), q_{4}\left(x_{4}\right)\right) \rightarrow q\left(c\left(x_{1}, x_{2}, x_{4}\right)\right)$. Then, the mbot $T_{a}$ obtained by decomposing $T$ contains the rules $b\left(q_{1}\left(x_{1}\right), q_{2}\left(x_{2}\right), q_{3}\left(x_{3}\right)\right) \rightarrow q_{1}^{\rho}\left(x_{1}, x_{2}\right)$ and $a\left(q_{1}^{\rho}\left(x_{1}, x_{2}\right), q_{4}\left(x_{4}\right)\right) \rightarrow q\left(c\left(x_{1}, x_{2}, x_{4}\right)\right)$ (See Fig. 3). Note that $q_{1}^{\rho}$ maintains $x_{1}$ and $x_{2}$ but not $x_{3}$ because $x_{3}$ does not occur in the right-hand side of $\rho$.

Lemma 7. Let $T_{a}=\left(Q_{a}, \Sigma, \Delta, Q_{f}, \delta_{a}\right)$ be the mbot obtained from an $x b o t^{-e}$ $T=\left(Q, \Sigma, \Delta, Q_{f}, \delta\right)$ by the above decomposition. Then, for every $q \in Q_{a}$ and $C \in \overline{\mathcal{C}}_{\Sigma}\left(\left\{x_{*}\right\}\right)$, if $C\left[q\left(x_{1}, \ldots, x_{k}\right)\right] \Rightarrow_{T_{a}}^{+} q\left(t_{1}, \ldots, t_{k}\right)$, then $\left(t_{1}, \ldots, t_{k}\right) \in \mathcal{T}_{\Delta}^{k, m}\left[X_{k}\right]$ for some $m \in[k]$.

Henceforth, we denote $q\left(t_{1}, \ldots, t_{k}\right)$ by $q(\mathbf{t})$ where $\mathbf{t}=\left(t_{1}, \ldots, t_{k}\right)$.

Lemma 8. Let $T_{a}=\left(Q_{a}, \Sigma, \Delta, Q_{f}, \delta_{a}\right)$ be the mbot obtained from an $x b o t^{-e} T$ by the above decomposition. Assume that $T_{a}$ has $n$ states and the maximum arity of states is $k_{m} . T_{a}$ is not single-valued if and only if there is a tree $t$ of depth less than $5 \cdot\left(n \cdot k_{m}\right)^{2}$ such that $\left|\llbracket T_{a} \rrbracket(t)\right|>1$. 


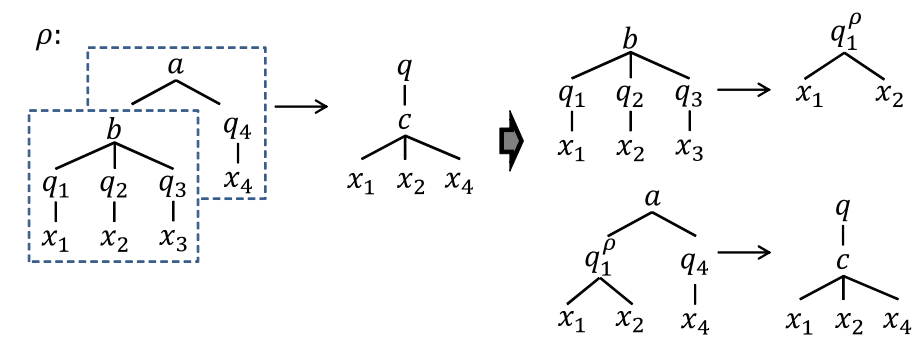

Fig. 3. An example of decomposing an $\mathrm{xbot}^{-e}$ to an mbot

Proof. The if part is trivial and so we prove the only if part. Assume that $t \in \mathcal{T}_{\Sigma}$ is a tree of minimal size such that there are two distinct derivations $t \Rightarrow_{T_{a}}^{*} q_{f 1}\left(t_{o 1}\right)$ and $t \Rightarrow_{T_{a}}^{*} q_{f 2}\left(t_{o 2}\right)$ where $q_{f 1}, q_{f 2} \in Q_{f}$, and $t_{o 1} \neq t_{o 2}$. For a contradiction, assume that the depth of $t$ is greater than or equal to $5 \cdot\left(n \cdot k_{m}\right)^{2}$. Then, by Lemma 7, there are two states $q_{1}, q_{2} \in Q_{a}$ with ranks $n_{1}$ and $n_{2}$ respectively, $C_{j} \in \overline{\mathcal{C}}_{\Sigma}\left(\left\{x_{*}\right\}\right)(0 \leq j \leq 4)$, $C_{5} \in \mathcal{T}_{\Sigma}$, and for $i \in\{1,2\}, m_{i} \in\left[n_{i}\right], t_{0}^{i} \in \mathcal{T}_{\Delta}\left[X_{n_{i}}\right]$, and $\mathbf{t}_{j}^{i} \in \mathcal{T}_{\Delta}^{n_{i}, m_{i}}\left[X_{n_{i}}\right](j \in[5])$ such that

$$
\begin{aligned}
t=C_{0} C_{1} C_{2} C_{3} C_{4} C_{5} & \Rightarrow T_{T_{a}}^{*} C_{0} C_{[1,4]}\left[q_{i}\left(\mathbf{t}_{5}^{i}\right)\right] \Rightarrow_{T_{a}}^{*} C_{0} C_{[1,3]}\left[q_{i}\left(\mathbf{t}_{[4,5]}^{i}\right)\right] \\
& \Rightarrow_{T_{a}}^{*} \cdots \\
& \Rightarrow_{T_{a}}^{*} C_{0}\left[q_{i}\left(\mathbf{t}_{[1,5]}^{i}\right)\right] \\
& \Rightarrow_{T_{a}}^{*} q_{f i}\left(t_{0}^{i} \mathbf{t}_{[1,5]}^{i}\right) .
\end{aligned}
$$

By the minimality of $t$, we have $t_{0}^{1} \mathbf{t}_{S}^{1}=t_{0}^{2} \mathbf{t}_{S}^{2} \in \llbracket T_{a} \rrbracket\left(C_{0} C_{S}\right)$ for every $S$ such that $\{5\} \subseteq S \subset[1,5]$. From Lemma $6, t_{o 1}=t_{0}^{1} \mathbf{t}_{[1,5]}^{1}=t_{0}^{2} \mathbf{t}_{[1,5]}^{2}=t_{o 2}$. This is a contradiction.

From Lemma 8, single-valuedness of $\operatorname{xbot}^{-e} \mathrm{~S}$ is decidable.

Theorem 1. It is decidable whether a given $x b^{-e}{ }^{-e}$ is single-valued. It is also decidable whether a given $x_{b o t}{ }^{+\mathrm{g}(\mathrm{B}(\mathrm{R}))}$ is single-valued.

Theorem 2. Determinacy is decidable for (sl-xbots, s-bots).

\subsection{Undecidability for Other Classes}

We show that determinacy is undecidable for (non-linear) $s$-bots as the determiner class. We prove the undecidability of determinacy for homomorphism tree transducers (homs) [11], which is a proper subclass of not only $s$-bots but also single-valued top-down tree transducers ( $s$-top). Let $i d$ be the class of the identity transductions on $\mathcal{T}_{\Sigma}$ for any alphabet $\Sigma$.

Theorem 3. Determinacy is undecidable for (homs, id). 
Proof. It can be shown by reduction from injectivity of homs, which is known to be undecidable [13]. Consider an arbitrary hom $T$. Then, it holds that $\llbracket T \rrbracket$ is injective if and only if $T$ determines the identity transducer $T_{i d}$.

Corollary 1. Determinacy is undecidable for (s-bots, id) and (s-tops, id).

Moreover, we have the undecidability result for deterministic monadic second-order logic defined tee transducers (dmsotts) [4,5], which is a proper superclass of $s l$-xbots.

Theorem 4. Determinacy is undecidable for (dmsotts, $\left.i d_{R}\right)$ where $i d_{R}$ is the class of the identities whose domains are regular tree languages.

Proof. It can be shown by reduction from ambiguity of context-free grammars. Consider an arbitrary context-free grammar $G$. Then, there is a dmsott $T_{G}$ which transforms any derivation tree of each string $s \in L(G)$ to $s$. Thus, $G$ is ambiguous if and only if $T_{G}$ determines the identity transducer $T_{i d}$ such that $\operatorname{dom}\left(T_{i d}\right)=\operatorname{dom}\left(T_{G}\right)$.

\section{Subsumption}

We show that subsumption is decidable for ( $s l$-xbots, $s$-bots). As shown in Section 3, given an $s l$-xbot $T_{1}$ and an $s$-bot $T_{2}$, if $T_{1}$ determines $T_{2}$, we can construct a reduced $s$-xbot $^{-e} T_{3}$ such that $\llbracket T_{3} \rrbracket=\llbracket T_{2} \rrbracket \circ \llbracket T_{1} \rrbracket^{-1}$. So, in order to decide subsumption, we should decide whether there is a bot equivalent with $T_{3}$. The next lemma provides a necessary and sufficient condition for an $s$ - $\operatorname{xbot}^{-e}$ to have an equivalent bot (see Appendix C).

Lemma 9. Let $T=\left(Q, \Sigma, \Delta, Q_{f}, \delta\right)$ be a reduced $s$-xbot ${ }^{-e}$. An s-bot equivalent with $T$ can be constructed if and only if $(\mathrm{X})$ for every rule $C_{l}\left[q_{1}\left(x_{1}\right), \ldots, q_{k}\left(x_{k}\right)\right] \rightarrow q\left(t_{r}\right) \in$ $\delta$ and any three variables $x_{i_{1}}, x_{i_{2}}, x_{i_{3}} \in \operatorname{var}\left(t_{r}\right)$, if

(X1) $\operatorname{rng}\left(T\left(q_{i_{j}}\right)\right)$ is infinite for all $j \in[3]$, and

(X2) nca $\left(p_{1}, p_{2}\right) \succ \operatorname{nca}\left(p_{1}, p_{3}\right)$ where $\left\{p_{j}\right\}=\operatorname{pos}_{x_{i_{j}}}\left(C_{l}\right)$ for $j \in[3]$, then

(X3) the minimal suffix $t_{s} \in \mathcal{T}_{\Sigma}\left(X_{k}\right)$ such that $t_{r}=t_{p} t_{s}$ for some $t_{p} \in$ $\overline{\mathcal{T}}_{\Sigma \cup X_{k}-\left\{x_{i_{1}}, x_{i_{2}}\right\}}\left(\left\{x_{*}\right\}\right)$ does not contain $x_{i_{3}}$.

Proof Sketch. Assume (X) does not hold and we can construct an s-bot $T^{\prime}$ equivalent with a given $s$-xbot ${ }^{-e} T$. Since (X) does not hold, there is a rule $C_{l}\left[q_{1}\left(x_{1}\right), \ldots, q_{k}\left(x_{k}\right)\right] \rightarrow q\left(t_{r}\right) \in \delta$ and $x_{i_{1}}, x_{i_{2}}, x_{i_{3}} \in \operatorname{var}\left(t_{r}\right)$ such that (X1) and (X2) hold but (X3) does not. Let $p_{12}=\operatorname{nca}\left(p_{1}, p_{2}\right)$ in (X2), and $t_{s}$ be the minimal suffix of $t_{r}$ in (X3). Since $T^{\prime}$ is an $s$-bot equivalent with $T, T^{\prime}$ must have rules of which left-hand sides 'cover' the subtree $\left.C_{l}\right|_{p_{12}}$, which contains $x_{i_{1}}$ and $x_{i_{2}}$ and does not contain $x_{i_{3}}$. Also, since $C_{l}\left[x_{*}\right]_{p_{12}}$ does not contain $x_{i_{1}}$ and $x_{i_{2}}$, some suffix $t_{s}^{\prime}$ of $t_{r}$ in the right-hand side such that $t_{r}=t_{p}^{\prime} t_{s}^{\prime}$ for some $t_{p}^{\prime} \in \overline{\mathcal{T}}_{\Sigma \cup X_{k}-\left\{x_{i_{1}}, x_{i_{2}}\right\}}\left(\left\{x_{*}\right\}\right)$ should be generated by $T^{\prime}$ corresponding to $\left.C_{l}\right|_{p_{12}}$. However, the minimal suffix $t_{s}$ contains $x_{i_{3}}$, and thus so does $t_{s}^{\prime}$. That is, $t_{s}^{\prime}$ including $x_{i_{3}}$ should be generated from $\left.C_{l}\right|_{p_{12}}$ without $x_{i_{3}}$, which leads a contradiction. Conversely, if (X) holds, we can divide each rule of $T$ into non-extended rules, each of which has exactly one symbol in the left-hand side.

For any xbot $T$, it can be decided whether $\operatorname{rng}(T)$ is infinite. Thus, it is decidable whether there is an $s$-bot equivalent with a given $s$-xbot ${ }^{-e}$.

Theorem 5. Subsumption is decidable for (sl-xbots, s-bots). 


\section{Conclusion}

We have shown that determinacy and subsumption are decidable for single-valued linear extended bottom-up tree transducers as the determiner class and single-valued bottom-up tree transducers as the determinee class. As for more powerful classes, we have shown that determinacy is undecidable for single-valued top-down/bottom-up tree transducers ( $s$-tops/bots) and deterministic MSO tree transducers (dmsotts) as the determiner class.

As future work, we will investigate whether subsumption for more powerful classes, such as $s$-tops/bots and dmsotts, is decidable or not. Though determinacy is undecidable for tops and dmsotts, decidability of subsumption for the classes is still open. We also consider whether, given two transducers $T_{1}$ and $T_{2}$ in the classes such that $T_{1}$ subsumes $T_{2}$, a transducer $T_{3}$ such that $\llbracket T_{2} \rrbracket=\llbracket T_{3} \rrbracket \circ \llbracket T_{1} \rrbracket$ can be effectively constructed or not.

\section{References}

1. Zheng, L., Chen, H.: Determinacy and rewriting of conjunctive queries over unary database schemas. In: Proceedings of the 2011 ACM Symposium on Applied Computing. (2011) 1039-1044

2. Afrati, F.N.: Determinacy and query rewriting for conjunctive queries and views. Theoretical Computer Science 412(11) (2011) 1005-1021

3. Fan, W., Geerts, F., Zheng, L.: View determinacy for preserving selected information in data transformations. Information Systems 37(1) (2012) 1-12

4. Courcelle, B.: Monadic second-order definable graph transductions: A survey. Theoretical Computer Science 126(1) (1994) 53-75

5. Engelfriet, J., Maneth, S.: The equivalence problem for deterministic MSO tree transducers is decidable. Information Processing Letters 100(5) (2006) 206-212

6. Fan, W., Bohannon, P.: Information preserving XML schema embedding. ACM Transactions on Database Systems 33(1) (2008) 4:1-4:44

7. Comon, H., Dauchet, M., Gilleron, R., Jacquemard, F., Lugiez, D., Löding, C., Tison, S., Tommasi, M.: Tree automata techniques and applications. http://www.grappa.univlille3.fr/tata (2007)

8. Lemay, A., Maneth, S., Niehren, J.: A learning algorithm for top-down XML transformations. In: Proceedings of the 29th ACM SIGMOD-SIGACT-SIGART symposium on Principles of database systems. (2010) 285-296

9. Engelfriet, J., Lilin, E., Maletti, A.: Extended multi bottom-up tree transducers. Acta Informatica 46 (2009) 561-590

10. Seidl, H.: Single-valuedness of tree transducers is decidable in polynomial time. Theoretical Computer Science 106(1) (1992) 135-181

11. Engelfriet, J.: Bottom-up and top-down tree transformations - a comparison. Mathematical Systems Theory 9(3) (1975) 198-231

12. Seidl, H.: Equivalence of finite-valued tree transducers is decidable. Theory of Computing Systems 27 (1994) 285-346

13. Fulop, Z., Gyenizse, P.: On injectivity of deterministic top-down tree transducers. Information Processing Letters 48(4) (1993) 183-188 


\section{A Construction of a Reduced xbot}

It is known that for every bot $T$ an equivalent reduced bot can be constructed from $T$ in linear time [10]. For xbots and $\mathrm{xbot}^{+\mathrm{g}_{\mathrm{S}}}$ defined in Section 3, equivalent reduced ones can also be constructed in the same way. Here, we recall the construction: Given xbot $^{+\mathrm{g}} T=\left(Q, \Sigma, \Delta, Q_{f}, \delta\right)$, to satisfy condition 2 for the reducedness in Section 2.2, first construct $T^{\prime}=\left(Q \times\{0,1\}, \Sigma, \Delta, Q_{f} \times\{1\}, \delta^{\prime}\right)$ such that for each rule $C_{l}\left[q_{1}\left(x_{1}\right), \ldots, q_{k}\left(x_{k}\right)\right] \rightarrow q\left(\tilde{t}_{r}\right) \in \delta$,

- $C_{l}\left[\hat{q}_{1}\left(x_{1}\right), \ldots, \hat{q}_{k}\left(x_{k}\right)\right] \rightarrow(q, 1)\left(\tilde{t}_{r}\right) \in \delta^{\prime}$ where $\hat{q}_{i}=\left(q_{i}, 1\right)$ if $x_{i}$ appears in $\tilde{t}_{r}$, and $\hat{q}_{i}=\left(q_{i}, 0\right)$ otherwise; and

- $C_{l}\left[\left(q_{1}, 0\right)\left(x_{1}\right), \ldots,\left(q_{k}, 0\right)\left(x_{k}\right)\right] \rightarrow(q, 0)(\perp) \in \delta^{\prime}$.

Next, for condition 3 for the reducedness, add a new state $q^{f}$ and replace the set of final states with the singleton $\left\{q^{f}\right\}$. Then for any rule $\rho \in \delta^{\prime}$ whose right-hand side has a state $\hat{q} \in Q_{f} \times\{1\}$, add the new rule obtained from $\rho$ by replacing $\hat{q}$ with $q^{f}$ in the right-hand side. Now, the reduced $x b^{+g} T^{\prime \prime}$ equivalent with $T$ is obtained by removing useless states for condition 1 of the reducedness. Removing useless states can be done in the same way as for tree automata [7]. Notice that $U\left(T^{\prime \prime}\right)=Q^{\prime \prime} \cap(Q \times\{0\})$ where $Q^{\prime \prime}$ is the set of states of $T^{\prime \prime}$.

\section{B Proofs of Lemmas for Theorem 1}

\section{B.1 Proof of Lemma 6}

We first recall the notations. Let $\mathcal{T}_{\Sigma}\left[X_{n}\right]=\overline{\mathcal{T}}_{\Sigma}\left(X_{n}\right) \cup \mathcal{T}_{\Sigma}$, that is, every $t \in \mathcal{T}_{\Sigma}\left[X_{n}\right]$ has all the variables in $X_{n}$ or has no variable. For $t, s \in \mathcal{T}_{\Sigma}\left[X_{n}\right]$, ts is the tree obtained from $t$ by replacing each variable with $s$. Note that $t s=t$ if $t$ has no variable. For $m \in[n]$, let $\mathcal{T}_{\Sigma}^{n, m}\left[X_{n}\right]=\mathcal{T}_{\Sigma}^{m-1} \times \mathcal{T}_{\Sigma}\left[X_{n}\right] \times \mathcal{T}_{\Sigma}^{n-m}$. For $\mathbf{t} \in \mathcal{T}_{\Sigma}^{n, m}\left[X_{n}\right]$, we denote by $t^{(i)}$ the $i$ th element of $\mathbf{t}$, i.e., $\mathbf{t}=\left(t^{(1)}, \ldots, t^{(n)}\right)$. For $s \in \mathcal{T}_{\Sigma}\left[X_{n}\right]$ and $\mathbf{t} \in \mathcal{T}_{\Sigma}^{n, m}\left[X_{n}\right]$, st is the tree obtained from $s$ by replacing $x_{i}$ with $t^{(i)}$ for all $i \in[n]$. Let $\mathbf{t} s=\left(t^{(1)} s, \ldots, t^{(n)} s\right)$, and $\mathbf{t u}=\left(t^{(1)} \mathbf{u}, \ldots, t^{(n)} \mathbf{u}\right)$ for $\mathbf{u} \in \mathcal{T}_{\Sigma}^{n, m}\left[X_{n}\right]$. Notice that since $\mathbf{t} \in \mathcal{T}_{\Sigma}^{n, m}\left[X_{n}\right]$, so are $\mathbf{t} s$ and tu. Lastly, the above substitution operation is associative. That is, for $s \in \mathcal{T}_{\Sigma}\left[X_{n}\right]$ and $\mathbf{t}_{1}, \mathbf{t}_{2}, \mathbf{t}_{3} \in \mathcal{T}_{\Sigma}^{n, m}\left[X_{n}\right],\left(s \mathbf{t}_{1}\right) \mathbf{t}_{2}=s\left(\mathbf{t}_{1} \mathbf{t}_{2}\right)$ and $\left(\mathbf{t}_{1} \mathbf{t}_{2}\right) \mathbf{t}_{3}=\mathbf{t}_{1}\left(\mathbf{t}_{2} \mathbf{t}_{3}\right)$ hold. For $\mathbf{t}_{1}, \mathbf{t}_{2}, \mathbf{t}_{3}, \mathbf{t}_{4}, \mathbf{t}_{5} \in \mathcal{T}_{\Sigma}^{n, m}\left[X_{n}\right]$ and $S=\left\{i_{1}, \ldots, i_{|S|}\right\} \subseteq[1,5]$, let $\mathbf{t}_{S}=\mathbf{t}_{i_{1}} \cdots \mathbf{t}_{i_{|S|}}$ where $i_{j}<i_{j+1}$ for $j \in[|S|-1]$.

We use some propositions to prove Lemma 6. The following is the same as the top cancellation in [12] except that we use tuples of trees in $\mathcal{T}_{\Sigma}^{n, m}\left[X_{n}\right]$.

Proposition 2 (Top Cancellation). Let $s \in \mathcal{T}_{\Sigma}\left[X_{n}\right]$ and $\mathbf{t}_{1}, \mathbf{t}_{2} \in \mathcal{T}_{\Sigma}^{n, m}\left[X_{n}\right]$. If st $\mathbf{t}_{1}=$ $s \mathbf{t}_{2}$, then $s \in \mathcal{T}_{\Sigma}$ or $\mathbf{t}_{1}=\mathbf{t}_{2}$.

Proof. Assume that $s \mathbf{t}_{1}=s \mathbf{t}_{2}, s \notin \mathcal{T}_{\Sigma}$ and $\mathbf{t}_{1} \neq \mathbf{t}_{2}$. Then, $s$ contains all the variables in $X_{n}$, and $t_{1}^{(i)} \neq t_{2}^{(i)}$ for some $i \in[n]$. Since distinct trees are substituted to $x_{i}$, $s \mathbf{t}_{1} \neq s \mathbf{t}_{2}$. This is a contradiction. 
The decidability of single-valuedness (or more generally, $k$-valuedness) of bots was proved in [12] by using Engelfriet's Property T1(i), which was proved by Engelfriet's Property T2(i).

Proposition 3 (Engelfriet's Property T1(i) [12]). Assume $t_{i}, t_{i}^{\prime} \in \mathcal{T}_{\Sigma}\left(\left\{x_{*}\right\}\right), i=$ $0,1,2,3,4$. Then,

$$
t_{0} \cdots t_{i-1} t_{j} \cdots t_{4}=t_{0}^{\prime} \cdots t_{i-1}^{\prime} t_{j}^{\prime} \cdots t_{4}^{\prime} \quad \text { for all } 0<i<j \leq 4
$$

implies $t_{0} t_{1} t_{2} t_{3} t_{4}=t_{0}^{\prime} t_{1}^{\prime} t_{2}^{\prime} t_{3}^{\prime} t_{4}^{\prime}$.

Proposition 4 (Engelfriet's Property T2(i) [12]). Assume $s_{i}, u_{i}, v_{i}, y_{i}, z_{i} \in$ $\mathcal{T}_{\Sigma}\left(\left\{x_{*}\right\}\right)(i=1,2), s_{1}$ or $s_{2}$ contains $x_{*}, y_{1} \neq z_{1}$, and $y_{2} \neq z_{2}$. Then,

$$
\begin{aligned}
& s_{1} y_{1}=s_{2} y_{2} \\
& s_{1} z_{1}=s_{2} z_{2} \\
& s_{1} v_{1}=s_{2} v_{2} \quad \text { implies } \quad u_{1} v_{1}=u_{2} v_{2} . \\
& u_{1} y_{1}=u_{2} y_{2} \\
& u_{1} z_{1}=u_{2} z_{2}
\end{aligned}
$$

Now, we prove Lemma 6, a variant of Engelfriet's Property T1(i). For $\mathbf{t}=$ $\left(t^{(1)}, \ldots, t^{(n)}\right) \in \mathcal{T}_{\Sigma}^{n, m}\left[X_{n}\right], \mathbf{t}^{c}$ denotes the $n$-tuple of trees obtained from $\mathbf{t}$ by replacing the $m$ th element with $x_{*}$, that is, $\mathbf{t}^{c}=\left(t^{(1)}, \ldots, t^{(m-1)}, x_{*}, t^{(m+1)}, \ldots, t^{(n)}\right)$. Hence, we have $\mathbf{t}=\mathbf{t}^{c} t^{(m)}$.

Lemma 6. Let $n, n^{\prime}$ be arbitrary positive integers, and $m \in[n], m^{\prime} \in\left[n^{\prime}\right]$. Suppose that $t_{0} \in \mathcal{T}_{\Sigma}\left[X_{n}\right], \mathbf{t}_{1}, \mathbf{t}_{2}, \mathbf{t}_{3}, \mathbf{t}_{4}, \mathbf{t}_{5} \in \mathcal{T}_{\Sigma}^{n, m}\left[X_{n}\right], t_{0}^{\prime} \in \mathcal{T}_{\Sigma}\left[X_{n^{\prime}}\right], \mathbf{t}_{1}^{\prime}, \mathbf{t}_{2}^{\prime}, \mathbf{t}_{3}^{\prime}, \mathbf{t}_{4}^{\prime}, \mathbf{t}_{5}^{\prime} \in$ $\mathcal{T}_{\Sigma}^{n^{\prime}, m^{\prime}}\left[X_{n^{\prime}}\right]$. Then, if $t_{0} \mathbf{t}_{S}=t_{0}^{\prime} \mathbf{t}_{S}^{\prime}$ for every $S$ such that $\{5\} \subseteq S \subset[1,5]$, then $t_{0} \mathbf{t}_{[1,5]}=t_{0}^{\prime} \mathbf{t}_{[1,5]}^{\prime}$.

Proof. Assume that $t_{0} \mathbf{t}_{S}=t_{0}^{\prime} \mathbf{t}_{S}^{\prime}$ for every $S$ such that $\{5\} \subseteq S \subset[1,5]$. Let

$$
\begin{array}{ll}
s_{1}=t_{0} \mathbf{t}_{2}^{c}, & s_{2}=t_{0}^{\prime} \mathbf{t}_{2}^{\prime c}, \\
u_{1}=t_{0} \mathbf{t}_{1} \mathbf{t}_{2}^{c}, & u_{2}=t_{0}^{\prime} \mathbf{t}_{1}^{\prime} \mathbf{t}_{2}^{\prime c}, \\
y_{1}=t_{2}^{(m)} \mathbf{t}_{5}, & y_{2}=t_{2}^{\left(m^{\prime}\right)} \mathbf{t}_{5}^{\prime}, \\
z_{1}=t_{2}^{(m)} \mathbf{t}_{[4,5]}, & z_{2}=t_{2}^{\prime\left(m^{\prime}\right)} \mathbf{t}_{[4,5]}^{\prime}, \\
v_{1}=t_{2}^{(m)} \mathbf{t}_{[3,5]}, & v_{2}=t_{2}^{\prime\left(m^{\prime}\right)} \mathbf{t}_{[3,5]}^{\prime} .
\end{array}
$$

By the assumption, we have

$$
\begin{aligned}
s_{1} y_{1} & =\left(t_{0} \mathbf{t}_{2}^{c}\right)\left(t_{2}^{(m)} \mathbf{t}_{5}\right)=t_{0} \mathbf{t}_{2} \mathbf{t}_{5}=t_{0}^{\prime} \mathbf{t}_{2}^{\prime} \mathbf{t}_{5}^{\prime}=\left(t_{0}^{\prime} \mathbf{t}_{2}^{\prime c}\right)\left(t_{2}^{\prime\left(m^{\prime}\right)} \mathbf{t}_{5}^{\prime}\right)=s_{2} y_{2}, \\
s_{1} z_{1} & =\left(t_{0} \mathbf{t}_{2}^{c}\right)\left(t_{2}^{(m)} \mathbf{t}_{[4,5]}\right)=t_{0} \mathbf{t}_{2} \mathbf{t}_{[4,5]}=t_{0}^{\prime} \mathbf{t}_{2}^{\prime} \mathbf{t}_{[4,5]}^{\prime}=\left(t_{0}^{\prime} \mathbf{t}_{2}^{\prime c}\right)\left(t_{2}^{\prime\left(m^{\prime}\right)} \mathbf{t}_{[4,5]}^{\prime}\right)=s_{2} z_{2}, \\
s_{1} v_{1} & =\left(t_{0} \mathbf{t}_{2}^{c}\right)\left(t_{2}^{(m)} \mathbf{t}_{[3,5]}\right)=t_{0} \mathbf{t}_{[2,5]}=t_{0}^{\prime} \mathbf{t}_{[2,5]}^{\prime}=\left(t_{0}^{\prime} \mathbf{t}_{2}^{\prime c}\right)\left(t_{2}^{\prime\left(m^{\prime}\right)} \mathbf{t}_{[3,5]}^{\prime}\right)=s_{2} v_{2}, \\
u_{1} y_{1} & =\left(t_{0} \mathbf{t}_{1} \mathbf{t}_{2}^{c}\right)\left(t_{2}^{(m)} \mathbf{t}_{5}\right)=t_{0} \mathbf{t}_{1} \mathbf{t}_{2} \mathbf{t}_{5}=t_{0}^{\prime} \mathbf{t}_{1}^{\prime} \mathbf{t}_{2}^{\prime} \mathbf{t}_{5}^{\prime}=\left(t_{0}^{\prime} \mathbf{t}_{1}^{\prime} \mathbf{t}_{2}^{\prime c}\right)\left(t_{2}^{\prime\left(m^{\prime}\right)} \mathbf{t}_{5}^{\prime}\right)=u_{2} y_{2}, \\
u_{1} z_{1} & =\left(t_{0} \mathbf{t}_{1} \mathbf{t}_{2}^{c}\right)\left(t_{2}^{(m)} \mathbf{t}_{[4,5]}\right)=t_{0} \mathbf{t}_{[1,2]} \mathbf{t}_{[4,5]}=t_{0}^{\prime} \mathbf{t}_{[1,2]}^{\prime} \mathbf{t}_{[4,5]}^{\prime}=\left(t_{0}^{\prime} \mathbf{t}_{1}^{\prime} \mathbf{t}_{2}^{\prime c}\right)\left(t_{2}^{\prime\left(m^{\prime}\right)} \mathbf{t}_{[4,5]}^{\prime}\right) \\
& =u_{2} z_{2} . \\
u_{1} v_{1} & =\left(t_{0} \mathbf{t}_{1} \mathbf{t}_{2}^{c}\right)\left(t_{2}^{(m)} \mathbf{t}_{[3,5]}\right)=t_{0} \mathbf{t}_{[1,5]} \\
u_{2} v_{2} & =\left(t_{0}^{\prime} \mathbf{t}_{1}^{\prime} \mathbf{t}_{2}^{\prime c}\right)\left(t_{2}^{\prime\left(m^{\prime}\right)} \mathbf{t}_{[3,5]}^{\prime}\right)=t_{0}^{\prime} \mathbf{t}_{[1,5]}^{\prime}
\end{aligned}
$$


There are four cases.

Case (1) Both of $s_{1}$ and $s_{2}$ contain no variable.

Then, $t_{0}$ and $t_{0}^{\prime}$ do not contain $x_{m}$ and $x_{m^{\prime}}$, respectively. Moreover, by definition of $\mathcal{T}_{\Sigma}[X], t_{0}$ and $t_{0}^{\prime}$ do not contain any variable. Thus, $t_{0} \mathbf{t}_{[1,5]}=t_{0}=t_{0} \mathbf{t}_{5}$ and $t_{0}^{\prime} \mathbf{t}_{[1,5]}^{\prime}=$ $t_{0}^{\prime}=t_{0}^{\prime} \mathbf{t}_{5}^{\prime}$. Since $t_{0} \mathbf{t}_{5}=t_{0}^{\prime} \mathbf{t}_{5}^{\prime}$, we have $t_{0} \mathbf{t}_{[1,5]}=t_{0}^{\prime} \mathbf{t}_{[1,5]}$.

Case (2) $s_{1}$ or $s_{2}$ contains variable $x_{*}, y_{1} \neq z_{1}$ and $y_{2} \neq z_{2}$.

Proposition 4 implies $t_{0} \mathbf{t}_{[1,5]}=u_{1} v_{1}=u_{2} v_{2}=t_{0}^{\prime} \mathbf{t}_{[1,5]}$.

Case (3) $s_{1}$ or $s_{2}$ contains variable $x_{*}$ and $y_{1}=z_{1}$.

Because $y_{1}=z_{1}$, by top cancellation (Proposition 2), $\mathbf{t}_{5}=\mathbf{t}_{[4,5]}$ or $t_{2}^{(m)}$ has no variable. If $\mathbf{t}_{5}=\mathbf{t}_{[4,5]}$ then $t_{0} \mathbf{t}_{[1,3]} \mathbf{t}_{5}=t_{0} \mathbf{t}_{[1,5]}$. If $t_{2}^{(m)}$ has no variable then we also have $t_{0} \mathbf{t}_{[1,3]} \mathbf{t}_{5}=t_{0} \mathbf{t}_{[1,2]}=t_{0} \mathbf{t}_{[1,5]}$. Moreover, we have $s_{1} y_{1}=s_{1} z_{1}$ and thus $s_{2} y_{2}=s_{2} z_{2}$. By top cancellation, $y_{2}=z_{2}$ or $s_{2}$ has no variable. Assume that $y_{2}=z_{2}$. By the same argument as the above, we have $t_{0}^{\prime} \mathbf{t}_{[1,3]}^{\prime} \mathbf{t}_{5}^{\prime}=t_{0}^{\prime} \mathbf{t}_{[1,5]}^{\prime}$. On the other hand, assume that $s_{2}$ has no variables. Then, $t_{0}^{\prime}$ has no variable and thus we also have $t_{0}^{\prime} \mathbf{t}_{[1,3]}^{\prime} \mathbf{t}_{5}^{\prime}=t_{0}^{\prime}=t_{0}^{\prime} \mathbf{t}_{[1,5]}^{\prime}$. Therefore, $t_{0} \mathbf{t}_{[1,5]}=t_{0}^{\prime} \mathbf{t}_{[1,5]}^{\prime}$ because $t_{0} \mathbf{t}_{[1,3]} \mathbf{t}_{5}=t_{0}^{\prime} \mathbf{t}_{[1,3]}^{\prime} \mathbf{t}_{5}^{\prime}$. Case (4) $s_{1}$ or $s_{2}$ contains variable $x_{*}$ and $y_{2}=z_{2}$.

This is analogous to Case (3).

\section{B.2 Proof of Lemma 7}

To decompose the left-hand side of each rule of a given $\mathrm{xbot}^{-e}$ into several rules each of which has only one input symbol, we construct a multi bottom-up tree transducer equivalent with the $\mathrm{xbot}^{-e}$. A multi bottom-up tree transducer [9] is a bottom-up tree transducer whose states might have ranks different from one. A multi bottom-up tree transducer (mbot) is a 5-tuple $\left(Q, \Sigma, \Delta, Q_{f}, \delta\right)$ where $Q_{f} \subseteq Q^{(1)}$ and $\delta$ is a set of rules of the form $l \rightarrow r$ where $l \in \Sigma(Q(X))$ is linear in $X$ and $r \in Q\left(\mathcal{T}_{\Delta}(\operatorname{var}(l))\right)$. For $q \in Q^{(k)}$ and a finite subset $X_{q}=\left\{x_{i_{1}}, \ldots, x_{i_{k}}\right\}$ of $X-\left\{x_{*}\right\}$, let $q\left(X_{q}\right)$ denote $q\left(x_{i_{1}}, \ldots, x_{i_{k}}\right)$ where $i_{j}<i_{j+1}$ for every $j \in[k-1]$. The move relation $\Rightarrow_{T}$ of a mbot $T=\left(Q, \Sigma, \Delta, Q_{f}, \delta\right)$ is defined as follows: $t \Rightarrow_{T} t^{\prime}$ if there is a rule $l \rightarrow r \in \delta$, $p \in \operatorname{pos}(t)$, and a substitution $\theta: X \rightarrow \mathcal{T}_{\Sigma \cup \Delta}$ such that $\left.t\right|_{p}=l \theta$ and $t^{\prime}=t[r \theta]_{p}$.

The decomposition is as follows: Given an $\mathrm{xbot}^{-e} T=\left(Q, \Sigma, \Delta, Q_{f}, \delta\right)$, construct an mbot $T_{\mathrm{m}}=\left(Q \cup Q_{\mathrm{m}}, \Sigma, \Delta, Q_{f}, \delta_{\mathrm{m}}\right)$ where

- $Q_{\mathrm{m}}=\left\{q_{p}^{\rho} \mid \rho=\left(C_{l}\left[q_{1}\left(x_{1}\right), \ldots, q_{k}\left(x_{k}\right)\right] \rightarrow q\left(t_{r}\right)\right) \in \delta, p \in \operatorname{pos}_{\Sigma}\left(C_{l}\right)-\{\epsilon\}\right\}$,

- $\delta_{\mathrm{m}}$ is the smallest set such that for each $\rho=\left(C_{l}\left[q_{1}\left(x_{1}\right), \ldots, q_{k}\left(x_{k}\right)\right] \rightarrow q\left(t_{r}\right)\right) \in \delta$, - $\sigma_{\epsilon}\left(q_{1}^{\rho}\left(V_{1}\right), \ldots, q_{k_{\epsilon}}^{\rho}\left(V_{k_{\epsilon}}\right)\right) \rightarrow q\left(t_{r}\right) \in \delta_{\mathrm{m}}$ where $\sigma_{\epsilon}=\lambda_{C_{l}}(\epsilon), k_{\epsilon}=\mathrm{rk}\left(\sigma_{\epsilon}\right)$, and $V_{i}=\operatorname{var}\left(\left.C_{l}\right|_{i}\right) \cap \operatorname{var}\left(t_{r}\right)$ for each $i \in\left[k_{\epsilon}\right]$,

- for each position $p \in \operatorname{pos}_{\Sigma}\left(C_{l}\right)-\{\epsilon\}$, let $V_{p}=\operatorname{var}\left(\left.C_{l}\right|_{p}\right)$ and $\theta_{n}: V_{p} \rightarrow X_{\left|V_{p}\right|}$ such that $\left.C_{l}\right|_{p} \theta_{n}$ is normalized, then the rule obtained by normalizing the both sides of $\sigma_{p}\left(q_{p 1}^{\rho}\left(V_{p 1}\right), \ldots, q_{p k_{p}}^{\rho}\left(V_{p k_{p}}\right)\right) \rightarrow q_{p}^{\rho}\left(V_{p}\right)$ by $\theta_{n}$ belongs to $\delta_{\mathrm{m}}$, where $\sigma_{p}=\lambda_{C_{l}}(p), k_{p}=\operatorname{rk}\left(\sigma_{p}\right)$, and $V_{p i}=\operatorname{var}\left(\left.C_{l}\right|_{p i}\right) \cap \operatorname{var}\left(t_{r}\right)$ for each $i \in\left[k_{p}\right]$, where $q_{p}^{\rho}=q_{i}$ if $p \in \operatorname{pos}_{x_{i}}\left(C_{l}\right)$ for some $x_{i} \in X_{k}$.

Lemma 7. Let $T_{\mathrm{m}}=\left(Q \cup Q_{\mathrm{m}}, \Sigma, \Delta, Q_{f}, \delta_{\mathrm{m}}\right)$ be the mbot obtained from an $x b^{-e}$ $T=\left(Q, \Sigma, \Delta, Q_{f}, \delta\right)$ by the above decomposition. Then, for every $q \in Q \cup Q_{\mathrm{m}}$ and $C \in \overline{\mathcal{C}}_{\Sigma}\left(\left\{x_{*}\right\}\right)$, if $C\left[q\left(x_{1}, \ldots, x_{k}\right)\right] \Rightarrow_{T_{\mathrm{m}}}^{+} q\left(t_{1}, \ldots, t_{k}\right)$, then $\left(t_{1}, \ldots, t_{k}\right) \in \mathcal{T}_{\Delta}^{k, m}\left[X_{k}\right]$ for some $m \in[k]$. 
Proof. Assume that $q \in Q$. Since every state in $Q$ has rank one, for any $C \in \overline{\mathcal{C}}_{\Sigma}\left(\left\{x_{*}\right\}\right)$, if $C\left[q\left(x_{*}\right)\right] \Rightarrow_{T_{\mathrm{m}}}^{*} q(t)$, then $t \in \mathcal{T}_{\Delta}\left(\left\{x_{*}\right\}\right)=\mathcal{T}_{\Delta}^{1,1}$.

Assume that $q \in Q_{\mathrm{m}}$ and $C$ is an arbitrary tree in $\overline{\mathcal{C}}_{\Sigma}\left(\left\{x_{*}\right\}\right)$ such that $C\left[q\left(x_{1}, \ldots, x_{k}\right)\right] \Rightarrow_{T_{\mathrm{m}}}^{+} q\left(t_{1}, \ldots, t_{k}\right)$. From the decomposition procedure, there exists a rule $\rho=\left(C_{l}\left[q_{1}\left(x_{1}\right), \ldots, q_{k}\left(x_{k}\right)\right] \rightarrow q_{\rho}\left(t_{r}\right)\right) \in \delta$ of $T$ such that $q=q_{p}^{\rho}$ for some position $p$ in $\operatorname{pos}_{\Sigma}\left(C_{l}\right)-\{\epsilon\}$. Thus, $C\left[q\left(x_{1}, \ldots, x_{k}\right)\right] \Rightarrow_{\rho}^{*} C^{\prime}\left[q_{\rho}\left(t^{\prime \prime}\right)\right] \Rightarrow_{T_{\mathrm{m}}}^{*} q\left(t_{1}, \ldots, t_{k}\right)$ for some $C^{\prime} \in \overline{\mathcal{C}}_{\Sigma}\left(\left\{x_{*}\right\}\right)$ and $t^{\prime \prime} \in \overline{\mathcal{T}}_{\Delta}\left(X_{k}\right)$, where $\Rightarrow_{\rho}^{*}$ means zero or more applications of only the rules obtained by decomposing $\rho$. Note that $t^{\prime \prime}$ must contain all the variables $x_{1}, \ldots, x_{k}$. In the above derivation $C^{\prime}\left[q_{\rho}\left(t^{\prime \prime}\right)\right] \Rightarrow_{T_{\mathrm{m}}}^{*} q\left(t_{1}, \ldots, t_{k}\right), t^{\prime \prime}$ is either abandoned or contained as a subtree in $t_{m}$ for some $m \in[k]$. Thus, $\left(t_{1}, \ldots, t_{k}\right) \in \mathcal{T}_{\Delta}^{k, m}$ for some $m \in[k]$.

\section{Proof of Lemma 9}

To prove Lemma 9, we use the following property, which is a slight extension of Proposition 1.5 in [10].

Proposition 5. Assume $t \in \mathcal{T}_{\Sigma}\left(X_{k}\right)$ contains at least one occurrence of each variable in $V \subseteq X_{k}$ where $|V| \geq 2$. Then, $t=t_{p} t_{s}$ for some $t_{p} \in \overline{\mathcal{T}}_{\Sigma \cup X_{k}-V}\left(\left\{x_{*}\right\}\right)$ and $t_{s} \in \mathcal{T}_{\Sigma}\left(X_{k}\right)$ satisfying the following conditions:

1. If $t=u_{p} u_{s}$ for some $u_{p} \in \overline{\mathcal{T}}_{\Sigma \cup X_{k}-V}\left(\left\{x_{*}\right\}\right)$ and $u_{s} \in \mathcal{T}_{\Sigma}\left(X_{k}\right)$ then $t_{p}=u_{p} r$ and $u_{s}=r t_{s}$ for some $r \in \overline{\mathcal{T}}_{\Sigma \cup X_{k}-V}\left(\left\{x_{*}\right\}\right)$. That is, $t_{p}$ and $t_{s}$ are the unique maximal prefix and minimal suffix of $t$, respectively.

2. Assume $t_{i} \in \overline{\mathcal{T}}_{\Sigma}\left(\left\{x_{i}\right\}\right)$ for each $x_{i} \in X_{k}$. Then, $t_{p}\left[t_{1}, \ldots, t_{k}\right] \in \overline{\mathcal{T}}_{\Sigma \cup X_{k}-V}\left(\left\{x_{*}\right\}\right)$ and $t_{s}\left[t_{1}, \ldots, t_{k}\right] \in \mathcal{T}_{\Sigma}\left(X_{k}\right)$ are the maximal prefix and the minimal suffix of $t\left[t_{1}, \ldots, t_{k}\right]$, respectively.

Lemma 9. Let $T=\left(Q, \Sigma, \Delta, Q_{f}, \delta\right)$ be a reduced s-xbot ${ }^{-e}$. An s-bot equivalent with $T$ can be constructed if and only if $(\mathrm{X})$ for every rule $C_{l}\left[q_{1}\left(x_{1}\right), \ldots, q_{k}\left(x_{k}\right)\right] \rightarrow q\left(t_{r}\right) \in$ $\delta$ and any three variables $x_{i_{1}}, x_{i_{2}}, x_{i_{3}} \in \operatorname{var}\left(t_{r}\right)$ if

(X1) $\operatorname{rng}\left(T\left(q_{i_{j}}\right)\right)$ is infinite for all $j \in[3]$, and

(X2) nca $\left(p_{1}, p_{2}\right) \succ \operatorname{nca}\left(p_{1}, p_{3}\right)$ where $\left\{p_{j}\right\}=\operatorname{pos}_{x_{i_{j}}}\left(C_{l}\right)$ for $j \in[3]$, then

(X3) the minimal suffix $t_{s} \in \mathcal{T}_{\Sigma}\left(X_{k}\right)$ such that $t_{r}=t_{p} t_{s}$ for some $t_{p} \in$ $\overline{\mathcal{T}}_{\Sigma \cup X_{k}-\left\{x_{i_{1}}, x_{i_{2}}\right\}}\left(\left\{x_{*}\right\}\right)$ does not contain $x_{i_{3}}$.

Proof. For the only if part, assume that (X) does not hold, i.e., there is a rule $\rho=$ $\left(C_{l}\left[q_{1}\left(x_{1}\right), \ldots, q_{k}\left(x_{k}\right)\right] \rightarrow q\left(t_{r}\right)\right) \in \delta$ that satisfies (X1) and (X2) but does not satisfy

(X3). That is, the rule $\rho$ has three variables $x_{i_{1}}, x_{i_{2}}, x_{i_{3}} \in \operatorname{var}\left(t_{r}\right)$ such that

- $\operatorname{rng}\left(T\left(q_{i_{j}}\right)\right)$ is infinite for all $j \in[3]$,

- nca $\left(p_{1}, p_{2}\right) \succ \operatorname{nca}\left(p_{1}, p_{3}\right)$ where $\left\{p_{j}\right\}=\operatorname{pos}_{x_{i_{j}}}\left(C_{l}\right)$ for $j \in[3]$, and

- the minimal suffix $t_{s 12} \in \mathcal{T}_{\Sigma}\left(X_{k}\right)$ such that $t_{r}=t_{p 12} t_{s 12}$ for some $t_{p 12} \in$ $\overline{\mathcal{T}}_{\Sigma \cup X_{k}-\left\{x_{i_{1}}, x_{i_{2}}\right\}}\left(\left\{x_{*}\right\}\right)$ contains $x_{i_{3}}$. 
For a contradiction, assume that there is a reduced $s$-bot $T^{s}=\left(Q^{s}, \Sigma, \Delta, Q_{f}^{s}, \delta^{s}\right)$ equivalent with $T$. Since $T$ is reduced and the three variables $x_{i_{1}}, x_{i_{2}}, x_{i_{3}}$ belong to $\operatorname{var}\left(t_{r}\right)$ of the rule $\rho$, we have that $q, q_{i_{1}}, q_{i_{2}}, q_{i_{3}} \in Q-U(T)$. Let $C_{l}^{\prime}=C_{l} \theta \in$ $\overline{\mathcal{C}}_{\Sigma}\left(X_{3}\right)$ such that $\theta\left(x_{i_{j}}\right)=x_{j}$ for $j \in[3]$ and $\theta\left(x_{i}\right)$ is a tree in $\operatorname{dom}\left(T\left(q_{i}\right)\right)$ for $x_{i} \in X_{k}-\left\{x_{i_{1}}, x_{i_{2}}, x_{i_{3}}\right\}$. Then,

$$
C C_{l}^{\prime}\left[q_{i_{1}}\left(x_{1}\right), q_{i_{2}}\left(x_{2}\right), q_{i_{3}}\left(x_{3}\right)\right] \Rightarrow_{T}^{*} C\left[q\left(t_{r}^{\prime}\right)\right] \Rightarrow_{T}^{*} q_{f}\left(t^{\prime} t_{r}^{\prime}\right)
$$

for some $C \in \overline{\mathcal{C}}_{\Sigma}\left(\left\{x_{*}\right\}\right), t^{\prime} \in \overline{\mathcal{T}}_{\Delta}\left(\left\{x_{*}\right\}\right), q_{f} \in Q_{f}$ and $t_{r}^{\prime}=t_{r} \theta^{\prime} \in \overline{\mathcal{T}}_{\Delta}\left(X_{3}\right)$ such that $\theta^{\prime}\left(x_{i_{j}}\right)=x_{j}$ for $j \in[3]$ and $\theta^{\prime}\left(x_{i}\right)=T\left(q_{i}\right)\left(\theta\left(x_{i}\right)\right)$ for $x_{i} \in X_{k}-\left\{x_{i_{1}}, x_{i_{2}}, x_{i_{3}}\right\}$. Since $T$ is single-valued, for each $j \in[3]$, there is an injective mapping $\xi_{j}$ : $\operatorname{rng}\left(T\left(q_{i_{j}}\right)\right) \rightarrow \operatorname{dom}\left(T\left(q_{i_{j}}\right)\right)$ such that $T\left(q_{i_{j}}\right)\left(\xi_{j}\left(t^{\prime}\right)\right)=t^{\prime}$ for any $t^{\prime} \in \operatorname{rng}\left(T\left(q_{i_{j}}\right)\right)$. Since $T^{s}$ is equivalent with $T$, for every $t_{1}^{\prime} \in \operatorname{rng}\left(T\left(q_{i_{1}}\right)\right), t_{2}^{\prime} \in \operatorname{rng}\left(T\left(q_{i_{2}}\right)\right)$, and $t_{3}^{\prime} \in \operatorname{rng}\left(T\left(q_{i_{3}}\right)\right)$, we have that $C C_{l}^{\prime}\left[\xi_{1}\left(t_{1}^{\prime}\right), \xi_{2}\left(t_{2}^{\prime}\right), \xi_{3}\left(t_{3}^{\prime}\right)\right] \Rightarrow_{T^{s}}^{*} q_{f}^{s}\left(t^{\prime} t_{r}^{\prime}\left[t_{1}^{\prime}, t_{2}^{\prime}, t_{3}^{\prime}\right]\right)$ for some $q_{f}^{s} \in Q_{s}$. By the fact that $\operatorname{rng}\left(T\left(q_{i_{j}}\right)\right)(j \in[3])$ is infinite but $Q_{s}$ is finite, and $\operatorname{nca}\left(p_{1}, p_{2}\right) \succ \operatorname{nca}\left(p_{1}, p_{3}\right)$, we can say that there are infinite sets $L_{1} \subseteq \operatorname{rng}\left(T\left(q_{i_{1}}\right)\right)$, $L_{2} \subseteq \operatorname{rng}\left(T\left(q_{i_{2}}\right)\right)$, and $L_{3} \subseteq \operatorname{rng}\left(T\left(q_{i_{3}}\right)\right)$, states $q_{1}^{s}, q_{2}^{s}, q_{3}^{s}, q_{12}^{s}, q^{s}, q_{f}^{s} \in Q_{s}$, and trees $u_{12}^{s} \in \mathcal{T}_{\Delta}\left(\left\{x_{1}, x_{2}\right\}\right), u_{3}^{s} \in \mathcal{T}_{\Delta}\left(\left\{x_{*}, x_{3}\right\}\right), u_{o}^{s} \in \mathcal{T}_{\Delta}\left(\left\{x_{*}\right\}\right)$ such that for all $t_{1}^{\prime} \in L_{1}$, $t_{2}^{\prime} \in L_{2}$, and $t_{3}^{\prime} \in L_{3}$,

$$
\begin{aligned}
& C C_{l}^{\prime}\left[\xi_{1}\left(t_{1}^{\prime}\right), \xi_{2}\left(t_{2}^{\prime}\right), \xi_{3}\left(t_{3}^{\prime}\right)\right] \\
& \Rightarrow_{T^{s}}^{*} C C_{l}^{\prime}\left[q_{1}^{s}\left(t_{1}^{s}\right), q_{2}^{s}\left(t_{2}^{s}\right), q_{3}^{s}\left(t_{3}^{s}\right)\right] \\
& \Rightarrow_{T^{s}}^{*} C\left(C_{l}^{\prime}\left[q_{12}^{s}\left(u_{12}^{s}\left[t_{1}^{s}, t_{2}^{s}\right]\right)\right]_{\mathrm{nca}\left(p_{1}, p_{2}\right)}\right)\left[x_{3} \leftarrow q_{3}^{s}\left(t_{3}^{s}\right)\right] \\
& \Rightarrow_{T^{s}}^{*} C\left[q^{s}\left(\left(u_{3}^{s} u_{12}^{s}\right)\left[t_{1}^{s}, t_{2}^{s}, t_{3}^{s}\right]\right)\right] \\
& \Rightarrow_{T^{s}}^{*} q_{f}^{s}\left(u_{o}^{s}\left(u_{3}^{s} u_{12}^{s}\right)\left[t_{1}^{s}, t_{2}^{s}, t_{3}^{s}\right]\right)
\end{aligned}
$$

and $u_{o}^{s}\left(u_{3}^{s} u_{12}^{s}\right)\left[t_{1}^{s}, t_{2}^{s}, t_{3}^{s}\right]=t^{\prime} t_{r}^{\prime}\left[t_{1}^{\prime}, t_{2}^{\prime}, t_{3}^{\prime}\right]$ where $\xi_{j}\left(t_{j}^{\prime}\right) \Rightarrow_{T^{s}}^{*} q_{j}^{s}\left(t_{j}^{s}\right)(j \in[3])$ (See Fig. 4). Now, fix $t_{2}^{\prime} \in L_{2}$ and $t_{3}^{\prime} \in L_{3}$. Consider $D_{1}=t^{\prime} t_{r}^{\prime}\left[x_{1}, t_{2}^{\prime}, t_{3}^{\prime}\right] \in \mathcal{T}_{\Delta}\left(\left\{x_{1}\right\}\right)$ and $D_{1}^{s}=u_{o}^{s}\left(u_{3}^{s} u_{12}^{s}\right)\left[x_{1}, t_{2}^{s}, t_{3}^{s}\right] \in \mathcal{T}_{\Delta}\left(\left\{x_{1}\right\}\right)$. Then, $L_{1}$ has two distinct trees $t_{1}^{\prime}$ and $t_{1}^{\prime \prime}$, and $D_{1}\left[t_{1}^{\prime}\right] \neq D_{1}\left[t_{1}^{\prime \prime}\right]$ because $q_{i_{1}} \notin U(T)$ and thus $D_{1}$ must have $x_{1}$. Since $T$ is single-valued and $T^{s}$ is equivalent with $T$, we have $D_{1}\left[t_{1}^{\prime}\right]=D_{1}^{s}\left[t_{1}^{\prime s}\right]$ and $D_{1}\left[t_{1}^{\prime \prime}\right]=D_{1}^{s}\left[t_{1}^{\prime \prime s}\right]$ where $\xi_{1}\left(t_{1}^{\prime}\right) \Rightarrow_{T^{s}}^{*} q_{1}^{s}\left(t_{1}^{s}\right)$ and $\xi_{1}\left(t_{1}^{\prime \prime}\right) \Rightarrow_{T^{s}}^{*} q_{1}^{s}\left(t_{1}^{\prime \prime s}\right)$. (Note that $t_{1}^{\prime s}$ and $t_{1}^{\prime \prime s}$ are uniquely determined respectively. If not, $T^{s}$ had two output trees for $C C_{l}^{\prime}\left[\xi_{1}\left(t_{1}^{\prime}\right), \xi_{2}\left(t_{2}^{\prime}\right), \xi_{3}\left(t_{3}^{\prime}\right)\right]$ and thus it was not single-valued. Since $T$ and $T_{s}$ are equivalent, however, $T^{s}$ is also single-valued. ) Therefore, $D_{1}^{s}$ also must have $x_{1}$, and there is a tree $r_{1}^{\prime} \in \overline{\mathcal{T}}_{\Delta}\left(\left\{x_{1}\right\}\right)$ such that $D_{1}\left[x_{1} \leftarrow r_{1}^{\prime}\right]=D_{1}^{s}$ or $D_{1}=D_{1}^{s}\left[x_{1} \leftarrow r_{1}^{\prime}\right]$. Similarly, for both $j=2,3$, there is a tree $r_{j}^{\prime} \in \overline{\mathcal{T}}_{\Delta}\left(\left\{x_{j}\right\}\right)$ such that $D_{j}\left[x_{j} \leftarrow r_{j}^{\prime}\right]=D_{j}^{s}$ or $D_{j}=D_{j}^{s}\left[x_{j} \leftarrow r_{j}^{\prime}\right]$. Thus, we have $t^{\prime} t_{r}^{\prime}\left[r_{1}, r_{2}, r_{3}\right]=u_{0}^{s}\left(u_{3}^{s} u_{12}^{s}\right)\left[r_{1}^{\prime \prime}, r_{2}^{\prime \prime}, r_{3}^{\prime \prime}\right]$ where $r_{j}$ and $r_{j}^{\prime \prime}$ are $r_{j}^{\prime}$ or $x_{j}$, and $r_{j}=x_{j}$ if and only if $r_{j}^{\prime \prime}=r_{j}^{\prime}$. By proposition 5 and $t^{\prime} t_{r}^{\prime}\left[r_{1}, r_{2}, r_{3}\right]=t^{\prime}\left(\left(t_{p 12} t_{s 12}\right) \theta^{\prime}\right)\left[r_{1}, r_{2}, r_{3}\right]$, we have $t_{s 12} \theta^{\prime}\left[r_{1}, r_{2}, r_{3}\right]$ is the minimal suffix of $t^{\prime} t_{r}^{\prime}\left[r_{1}, r_{2}, r_{3}\right]$ containing $x_{3}$. Thus, for any decomposition $t^{\prime} t_{r}^{\prime}\left[r_{1}, r_{2}, r_{3}\right]=t_{p} t_{s}, t_{s}$ must contain $x_{3}$. However, $u_{0}^{s}\left(u_{3}^{s} u_{12}^{s}\right)\left[r_{1}^{\prime \prime}, r_{2}^{\prime \prime}, r_{3}^{\prime \prime}\right]=$ $\left(u_{0}^{s} u_{3}^{s}\left[x_{3} \leftarrow r_{3}^{\prime \prime}\right]\right) u_{12}^{s}\left[r_{1}^{\prime \prime}, r_{2}^{\prime \prime}\right]$, and $u_{12}^{s}\left[r_{1}^{\prime \prime}, r_{2}^{\prime \prime}\right]$ does not contain $x_{3}$. This is a contradiction.

For the if part, assume that condition (X) holds. Let $Q_{\mathrm{fin}}=\{q \in Q \mid$ $\operatorname{rng}(T(q))$ is finite $\}$ and $Q_{\mathrm{fin}}^{n f}=Q_{\mathrm{fin}}-Q_{f}$. Note that $U(T)$ is included in $Q_{\mathrm{fin}}$ because 


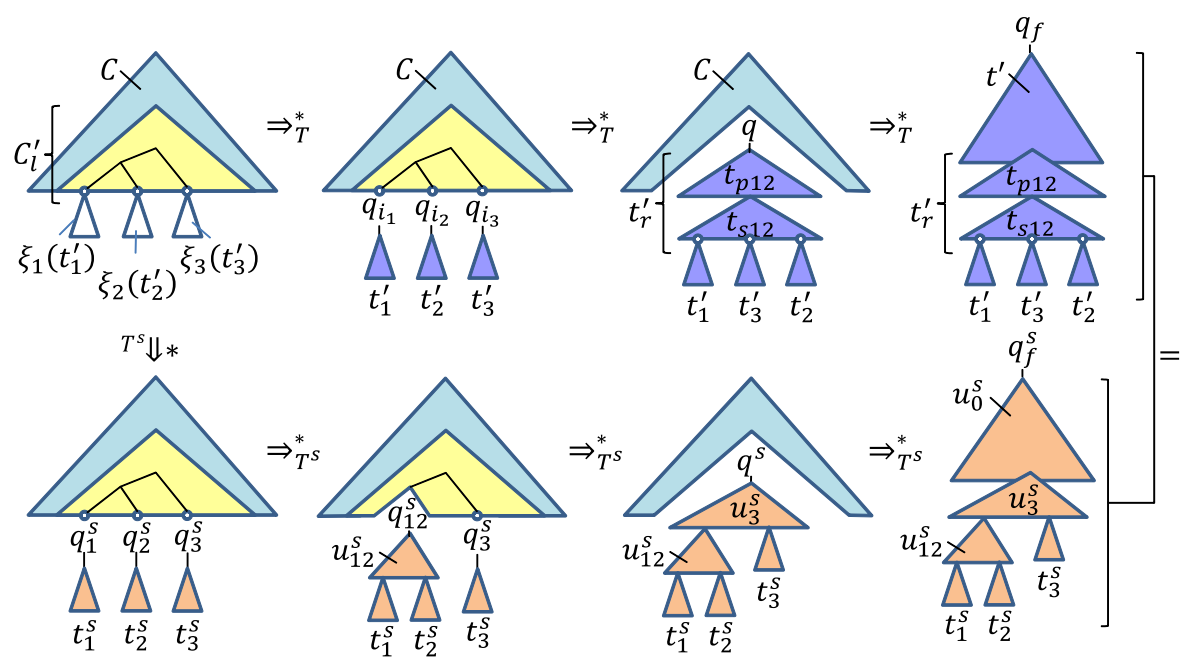

Fig. 4. Transduction by $T$ and $T^{s}$

$\operatorname{rng}(T(q))=\{\perp\}$ for $q \in U(T)$. Also, let $\tilde{Q}_{\text {fin }}^{n f}=\left\{(q, t) \mid q \in Q_{\text {fin }}^{n f}, t \in \operatorname{rng}(T(q))\right\}$. We first construct a reduced $s$-xbot ${ }^{-e} T_{f}$, equivalent with $T$, such that $\operatorname{rng}\left(T_{f}(q)\right)$ is infinite or the singleton $\{\perp\}$ for any non-final state $q \notin Q_{f}$ of $T_{f}$. More specifically, we construct $T_{f}=\left(\tilde{Q}_{\mathrm{fin}}^{n f} \cup\left(Q-Q_{\mathrm{fin}}^{n f}\right), \Sigma, \Delta, Q_{f}, \delta^{\prime}\right)$ such that $\delta^{\prime}$ is the smallest set satisfying the following condition: Let $C_{l}\left[q_{1}\left(x_{1}\right), \ldots, q_{k}\left(x_{k}\right)\right] \rightarrow q\left(t_{r}\right) \in \delta$ be an arbitrary rule.

- If $q \in Q_{\text {fin }}^{n f}$, then $C_{l}\left[\left(q_{1}, t_{1}\right)\left(x_{1}\right), \ldots,\left(q_{k}, t_{k}\right)\left(x_{k}\right)\right] \rightarrow\left(q, t_{r}\left[t_{1}, \ldots, t_{k}\right]\right)(\perp) \in \delta^{\prime}$ for all $t_{i} \in \operatorname{rng}\left(T\left(q_{i}\right)\right)(i \in[k])$.

- If $q \in Q-Q_{\mathrm{fin}}^{n f}$, let $\theta_{l}$ and $\theta_{r}$ be arbitrary substitutions such that for each $i \in[k]$, if $\operatorname{rng}\left(T\left(q_{i}\right)\right)$ is infinite, $\theta_{l}\left(x_{i}\right)=q_{i}\left(x_{i}\right)$ and $\theta_{r}\left(x_{i}\right)=x_{i}$; otherwise, $\theta_{l}\left(x_{i}\right)=$ $\left(q_{i}, t_{i}\right)\left(x_{i}\right)$ and $\theta_{r}\left(x_{i}\right)=t_{i}$ for any $t_{i} \in \operatorname{rng}\left(T\left(q_{i}\right)\right)$. Then, $C_{l} \theta_{l} \rightarrow q\left(t_{r} \theta_{r}\right) \in \delta^{\prime}$.

Note that $T_{f}$ is reduced and $U\left(T_{f}\right)=\tilde{Q}_{\text {fin }}^{n f}$. Thus, for every rule $C_{l}\left[q_{1}\left(x_{1}\right), \ldots, q_{k}\left(x_{k}\right)\right] \rightarrow q\left(t_{r}\right) \in \delta^{\prime}, x_{i} \in \operatorname{var}\left(t_{r}\right)$ if and only if $\operatorname{rng}\left(T_{f}\left(q_{i}\right)\right)$ is infinite for $i \in[k]$, that is, condition (X1) is satisfied. Namely,

For every $C_{l}\left[q_{1}\left(x_{1}\right), \ldots, q_{k}\left(x_{k}\right)\right] \rightarrow q\left(t_{r}\right) \in \delta^{\prime}$ and any three variables $x_{i_{1}}, x_{i_{2}}, x_{i_{3}} \in \operatorname{var}\left(t_{r}\right)$, if nca $\left(p_{1}, p_{2}\right) \succ \operatorname{nca}\left(p_{1}, p_{3}\right)$ where $\left\{p_{j}\right\}=\operatorname{pos}_{x_{i_{j}}}\left(C_{l}\right)$ for $j \in$ [3], then the minimal suffix $t_{s} \in \mathcal{T}_{\Sigma}\left(X_{k}\right)$ such that $t_{r}=t_{p} t_{s}$ for some $t_{p} \in \overline{\mathcal{T}}_{\Sigma \cup X_{k}-\left\{x_{i_{1}}, x_{i_{2}}\right\}}\left(\left\{x_{*}\right\}\right)$ does not contain $x_{i_{3}}$.

For $V \subseteq \operatorname{var}\left(t_{r}\right)$, let $\mathrm{dp}_{t_{r}}(V)=\operatorname{pos}_{x_{*}}\left(t_{p}\right)$ where $t_{p} \in \overline{\mathcal{T}}_{\Sigma \cup X_{k}-V}\left(\left\{x_{*}\right\}\right)$ is the maximal prefix of $t_{r}$ with respect to $V$ such that $t_{r}=t_{p} t_{s}$ for some $t_{s} \in \mathcal{T}_{\Sigma}\left(X_{k}\right)$.

Next, we decompose trees $C_{l}$ and $t_{r}$ in the both sides of each rule of $T_{f}$ with respect to the confluence positions of variables in $C_{l}$. A position $p$ of $C_{l}$ w.r.t $t_{r}$ is a confluence position if $\operatorname{var}\left(\left.C_{l}\right|_{p i}\right) \cap \operatorname{var}\left(t_{r}\right) \subsetneq \operatorname{var}\left(\left.C_{l}\right|_{p}\right) \cap \operatorname{var}\left(t_{r}\right)$ for all $i \in\left[\operatorname{rk}\left(\lambda_{C_{l}}(p)\right)\right]$. Let 


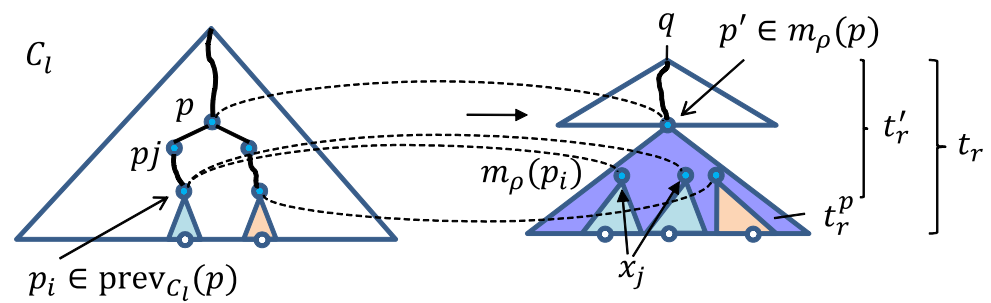

Fig. 5. Construction of $t_{r}^{p}$

$C P\left(C_{l}\right)$ be the set of all confluence positions of $C_{l}$. For $p \in C P\left(C_{l}\right)$, let $\operatorname{prev}_{C_{l}}(p)$ be the set of all the immediate previous confluence positions, that is, $\operatorname{prev}_{C_{l}}(p)=\left\{p^{\prime} \mid\right.$ $\left.p \prec p^{\prime}, \neg \exists p^{\prime \prime} \in C P\left(C_{l}\right): p \prec p^{\prime \prime} \prec p^{\prime}\right\}$.

By the assumption (X), for each rule $\rho=\left(C_{l}\left[q_{1}\left(x_{1}\right), \ldots, q_{k}\left(x_{k}\right)\right] \rightarrow q\left(t_{r}\right)\right) \in \delta^{\prime}$, there is a mapping $m_{\rho}: C P\left(C_{l}\right) \rightarrow 2^{\text {pos }\left(t_{r}\right)}$ such that $m_{\rho}(p)=\mathrm{dp}_{t_{r}}\left(\operatorname{var}\left(\left.C_{l}\right|_{p}\right)\right)$ for each $p \in C P\left(C_{l}\right)$. We can construct an $s$-bot $T^{\prime}=\left(\tilde{Q}_{\text {fin }}^{n f} \cup\left(Q-Q_{\text {fin }}^{n f}\right) \cup\right.$ $\left.Q_{m}, \Sigma, \Delta, Q_{f}, \delta^{\prime \prime}\right)$ from $T_{f}$ where

- $Q_{m}=\left\{q_{p}^{\rho} \mid \rho=\left(C_{l}\left[q_{1}\left(x_{1}\right), \ldots, q_{k}\left(x_{k}\right)\right] \rightarrow q\left(t_{r}\right)\right) \in \delta^{\prime}, p \in \operatorname{pos}_{\Sigma}\left(C_{l}\right)-\{\epsilon\}\right\}$,

- $\delta^{\prime \prime}$ is the smallest set such that for each rule $\rho=\left(C_{l}\left[q_{1}\left(x_{1}\right), \ldots, q_{k}\left(x_{k}\right)\right] \rightarrow\right.$ $\left.q\left(t_{r}\right)\right) \in \delta^{\prime}$,

- For each $p \in C P\left(C_{l}\right)$, let $\sigma_{p}=\lambda_{C_{l}}(p) \in \Sigma^{\left(k_{p}\right)}$, then

$$
\sigma_{p}\left(q_{p 1}^{\rho}\left(x_{1}\right), \ldots, q_{p k_{p}}^{\rho}\left(x_{k_{p}}\right)\right) \rightarrow q_{p}^{\rho}\left(t_{r}^{p}\right) \in \delta^{\prime \prime}
$$

where $t_{r}^{p}=\left.t_{r}^{\prime}\right|_{p^{\prime}}$ for any $p^{\prime} \in m_{\rho}(p)$ and $t_{r}^{\prime}$ is the tree obtained from $t_{r}$ by replacing all subtrees at positions in $m_{\rho}\left(p_{i}\right)$ with $x_{j}$ such that $p j \preceq p_{i}$ for all $p_{i} \in \operatorname{prev}_{C_{l}}(p)$ (See Fig. 5), and

- If $\epsilon \notin C P\left(C_{l}\right)$, let $\sigma_{\epsilon}=\lambda_{C_{l}}(\epsilon) \in \Sigma^{\left(k_{\epsilon}\right)}$, then

$$
\sigma_{\epsilon}\left(q_{1}^{\rho}\left(x_{1}\right), \ldots, q_{k_{\epsilon}}^{\rho}\left(x_{k_{\epsilon}}\right)\right) \rightarrow q_{\epsilon}^{\rho}\left(t_{r}^{\prime}\right) \in \delta^{\prime \prime}
$$

where $t_{r}^{\prime}$ is the tree obtained from $t_{r}$ by replacing all subtrees at positions in $\mathrm{dp}_{t_{r}}\left(\operatorname{var}\left(t_{r}\right)\right)$ with $x_{j}$ such that $\operatorname{var}\left(t_{r}\right) \subseteq \operatorname{var}\left(\left.C_{l}\right|_{j}\right)$.

- For each $p \in \operatorname{pos}_{\Sigma}\left(C_{l}\right)-\left(C P\left(C_{l}\right) \cup\{\epsilon\}\right)$, let $\sigma_{p}=\lambda_{C_{l}}(p) \in \Sigma^{\left(k_{p}\right)}$, then $* \sigma_{p}\left(q_{p 1}^{\rho}\left(x_{1}\right), \ldots, q_{p k_{p}}^{\rho}\left(x_{k_{p}}\right)\right) \rightarrow q_{p}^{\rho}\left(x_{i}\right) \in \delta^{\prime \prime}$ if $\left.C_{l}\right|_{p i}$ has at least one variable for some $i \in\left[k_{p}\right]$,

* $\sigma_{p}\left(q_{p 1}^{\rho}\left(x_{1}\right), \ldots, q_{p k_{p}}^{\rho}\left(x_{k_{p}}\right)\right) \rightarrow q_{p}^{\rho}(\perp) \in \delta^{\prime \prime}$ otherwise.

where $q_{\epsilon}^{\rho}=q$, and $q_{p}^{\rho}=q_{i}$ for each $x_{i} \in X_{k}$ and $p \in \operatorname{pos}_{x_{i}}\left(C_{l}\right)$.

$T^{\prime}$ is equivalent with $T_{f}$ because the rules obtained by decomposing each rule $\rho \in \delta^{\prime}$ can simulate $\rho$ exactly.

Therefore, condition (X) is a necessary and sufficient condition for an $s$-xbot ${ }^{-e}$ to have an equivalent $s$-bot $T$. 